\title{
THE GENERALIZED $k$-RESULTANT MODULUS SET PROBLEM IN FINITE FIELDS
}

\author{
DAVID COVERT, DOOWON KOH, AND YOUNGJIN PI
}

\begin{abstract}
Let $\mathbb{F}_{q}^{d}$ be the $d$-dimensional vector space over the finite field $\mathbb{F}_{q}$ with $q$ elements. Given $k$ sets $E_{j} \subset \mathbb{F}_{q}^{d}$ for $j=1,2, \ldots, k$, the generalized $k$-resultant modulus set, denoted by $\Delta_{k}\left(E_{1}, E_{2}, \ldots, E_{k}\right)$, is defined by

$\Delta_{k}\left(E_{1}, E_{2}, \ldots, E_{k}\right)=\left\{\left\|\mathbf{x}^{1}+\mathbf{x}^{2}+\cdots+\mathbf{x}^{k}\right\| \in \mathbb{F}_{q}: \mathbf{x}^{j} \in E_{j}, j=1,2, \ldots, k\right\}$, where $\|\mathbf{y}\|=\mathbf{y}_{1}^{2}+\cdots+\mathbf{y}_{d}^{2}$ for $\mathbf{y}=\left(\mathbf{y}_{1}, \ldots, \mathbf{y}_{d}\right) \in \mathbb{F}_{q}^{d}$. We prove that if $\prod_{j=1}^{3}\left|E_{j}\right| \geq C q^{3\left(\frac{d+1}{2}-\frac{1}{6 d+2}\right)}$ for $d=4,6$ with a sufficiently large constant $C>0$, then $\left|\Delta_{3}\left(E_{1}, E_{2}, E_{3}\right)\right| \geq c q$ for some constant $0<c \leq 1$, and if $\prod_{j=1}^{4}\left|E_{j}\right| \geq C q^{4\left(\frac{d+1}{2}-\frac{1}{6 d+2}\right)}$ for even $d \geq 8$, then $\left|\Delta_{4}\left(E_{1}, E_{2}, E_{3}, E_{4}\right)\right| \geq c q$. This generalizes the previous result in [5]. We also show that if $\prod_{j=1}^{3}\left|E_{j}\right| \geq$ $C q^{3\left(\frac{d+1}{2}-\frac{1}{9 d-18}\right)}$ for even $d \geq 8$, then $\left|\Delta_{3}\left(E_{1}, E_{2}, E_{3}\right)\right| \geq c q$. This result improves the previous work in [5] by removing $\varepsilon>0$ from the exponent.
\end{abstract}

\section{INTRODUCTION}

The Erdős distance problem asks us to determine the minimal number of distinct distances between any $N$ points in $\mathbb{R}^{d}$. This problem was initially posed by Paul Erdős [8] who conjectured that $g_{2}(N) \gtrsim N / \sqrt{\log N}$ and $g_{d}(N) \gtrsim N^{2 / d}$ for $d \geq 3$, where $g_{d}(N)$ denotes the minimal number of distinct distances between $N$ distinct points of $\mathbb{R}^{d}$, and $X \gtrsim Y$ for $X, Y>0$ means that there is a constant $C>0$ independent of $N$ such that $C X \geq Y$. This conjecture in two dimension was resolved up to the logarithmic factor by Guth and Katz [10]. However, the problem is still open in higher dimensions.

In [3], Bourgain, Katz, and Tao initially introduced the finite field analog of the Erdős distance problem and Iosevich and Rudnev [15] developed the problem in the general finite field setting. Let $\mathbb{F}_{q}^{d}$ be the $d$-dimensional vector space over the finite field $\mathbb{F}_{q}$ with $q$ elements. Throughout this paper we always assume that the characteristic of $\mathbb{F}_{q}$ is strictly greater than two. For a set $E \subset \mathbb{F}_{q}^{d}$, the distance set, denoted by $\Delta_{2}(E)$, is defined as

$$
\Delta_{2}(E)=\left\{\|\mathbf{x}-\mathbf{y}\| \in \mathbb{F}_{q}: \mathbf{x}, \mathbf{y} \in E\right\},
$$

2010 Mathematics Subject Classification. 52C10, 42B05, $11 \mathrm{~T} 23$.

Key words and phrases: Erdős distance problem, finite fields, $k$-resultant modulus set. Doowon Koh was supported by Basic Science Research Program through the National Research Foundation of Korea(NRF) funded by the Ministry of Education, Science and Technology(NRF2015R1A1A1A05001374). 
where $\|\alpha\|=\alpha_{1}^{2}+\cdots+\alpha_{d}^{2}$ for $\alpha=\left(\alpha_{1}, \ldots, \alpha_{d}\right) \in \mathbb{F}_{q}^{d}$. With this definition of the distance set, Bourgain, Katz, and Tao [3] proved that if $q \equiv 3(\bmod 4)$ is prime and $E \subset \mathbb{F}_{q}^{2}$ with $q^{\delta} \lesssim|E| \lesssim q^{2-\delta}$ for $\delta>0$, then there exists $\epsilon=\epsilon(\delta)$ such that $\left|\Delta_{2}(E)\right| \geq|E|^{1 / 2+\epsilon}$. Here we recall that $A \lesssim B$ for $A, B>0$ means that there exists a constant $C>0$ independent of $q$ such that $A \leq C B$, and we write $B \gtrsim A$ for $A \lesssim B$. We also use $A \sim B$ if $\lim _{q \rightarrow \infty} A / B=1$. This result was obtained by finding the connection between incidence geometry in $\mathbb{F}_{q}^{2}$ and the distance set. Unfortunately, it is not simple to find the relationship between $\delta$ and $\epsilon$ from their proof. Furthermore, if $E=\mathbb{F}_{q}^{2}$, then $\left|\Delta_{2}(E)\right|=\sqrt{|E|}$, which shows that the exponent $1 / 2$ can not be generically improved. If -1 is a square in $\mathbb{F}_{q}$, another unpleasant example exists with the finite field Erdős distance problem. For instance, let $E=\left\{(t, i t) \in \mathbb{F}_{q}^{2}: t \in \mathbb{F}_{q}\right\}$, where $i$ denotes an element of $\mathbb{F}_{q}$ such that $i^{2}=-1$. Then it is straightforward to see that $|E|=q$ and $\left|\Delta_{2}(E)\right|=|\{0\}|=1$. In view of aforementioned examples, Iosevich and Rudnev [15] reformulated the Erdős distance problem in general finite field setting as follows.

Question 1.1. Let $E \subset \mathbb{F}_{q}^{d}$. What is the smallest exponent $\beta>0$ such that if $|E| \geq C q^{\beta}$ for a sufficiently large constant $C>0$ then $\left|\Delta_{2}(E)\right| \geq c q$ for some $0<c \leq 1$ ?

The problem in this question is called the Erdös-Falconer distance problem in the finite field setting. Note that a distance can be viewed as an 1-dimensional simplex. Readers may refer to $[7,2,25,1,21]$ and references contained therein for the $k$-simplices problems. In [15], it was shown that $\beta \leq(d+1) / 2$ for all dimensions $d \geq 2$. The authors in [11] proved that $\beta=(d+1) / 2$ for general odd dimensions $d \geq 3$. On the other hand, they conjectured that if the dimension $d \geq 2$ is even, then $\beta$ can be improved to $d / 2$. In dimension two, the authors in [4] applied the sharp finite field restriction estimate for the circle on the plane so that they show $\beta \leq 4 / 3$ which improves the exponent $(d+1) / 2$, a sharp exponent in general odd dimensions $d$. It was also observed in [1] that the exponent $4 / 3$ can be obtained by applying the group action. Furthermore, considering the perpendicular bisector of two points in a set of $\mathbb{F}_{q}^{2}$, the authors in [12] proved that the exponent $4 / 3$ holds for the pinned distance problem case. However, in higher even dimensions $d \geq 4$, the exponent $(d+1) / 2$ has not been improved. To demonstrate some possibility that the exponent $(d+1) / 2$ could be improved for even dimensions $d \geq 4$, the authors in [5] introduced a $k$-resultant modulus set which generalizes the distance set in the sense that any $k$ points can be selected from a set $E \subset \mathbb{F}_{q}^{d}$ to determine an object similar to a distance. More precisely, for a set $E \subset \mathbb{F}_{q}^{d}$ we define a $k$-resultant modulus set $\Delta_{k}(E)$ as

$$
\Delta_{k}(E)=\left\{\left\|\mathbf{x}^{1} \pm \mathbf{x}^{2}+\cdots \pm \mathbf{x}^{k}\right\| \in \mathbb{F}_{q}: \mathbf{x}^{j} \in E\right\} .
$$

Since the sign " \pm " does not affect on our results in this paper, we shall simply take "+" signs. That is, we will use the definition

$$
\Delta_{k}(E)=\left\{\left\|\mathbf{x}^{1}+\mathbf{x}^{2}+\cdots+\mathbf{x}^{k}\right\| \in \mathbb{F}_{q}: \mathbf{x}^{j} \in E\right\}
$$

for consistency. With this definition of the $k$-resultant modulus set, the following question was proposed in [5]. 
Question 1.2. Let $E \subset \mathbb{F}_{q}^{d}$ and $k \geq 2$ be an integer. What is the smallest exponent $\gamma>0$ such that if $|E| \geq C q^{\gamma}$ for a sufficiently large constant $C>0$, then $\left|\Delta_{k}(E)\right| \geq$ cq for some $0<c \leq 1$ ?

This problem is called the $k$-resultant modulus problem. When $k=2$, this question is simply the finite field Erdös-Falconer distance problem, and in this sense the $k$-resultant modulus problem is a direct generalization of the distance problem. It is obvious that the smallest exponent $\beta>0$ in Question 1.1 is greater than or equal to the smallest exponent $\gamma>0$ in Question 1.2. In [5], it was conjectured that $\gamma$ must be equal to $\beta$. This conjecture means that the solution $\gamma$ of Question 1.2 is independent of the integer $k \geq 2$. In other words, it is conjectured that the solution of the Erdös-Falconer distance problem is the same as that of the $k$-resultant modulus problem. In fact, the authors in [6] provided a simple example which shows that $\gamma=\beta=(d+1) / 2$ for any integer $k \geq 2$ in odd dimensions $d \geq 3$ provided that -1 is a square number in $\mathbb{F}_{q}$. On the other hand, they conjectured that the smallest exponent $\gamma$ in Question 1.2 is $d / 2$ for even dimensions $d \geq 2$ and all integers $k \geq 2$. In addition, they showed that if $k \geq 3$ and the dimension $d$ is even, then one can improve the exponent $(d+1) / 2$ which is sharp in odd dimensional case. More precisely they obtained the following result.

Theorem 1.3. Let $E \subset \mathbb{F}_{q}^{d}$. Suppose that $C$ is a sufficiently large constant. Then the following statements hold:

(1) If $d=4$ or 6 , and $|E| \geq C q^{\frac{d+1}{2}-\frac{1}{6 d+2}}$, then $\left|\Delta_{3}(E)\right| \geq c q$ for some $0<c \leq 1$.

(2) If $d \geq 8$ is even and $|E| \geq C q^{\frac{d+1}{2}-\frac{1}{6 d+2}}$, then $\left|\Delta_{4}(E)\right| \geq c q$ for some $0<c \leq 1$.

(3) If $d \geq 8$ is even, then for any $\varepsilon>0$, there exists $C_{\varepsilon}>0$ such that if $|E| \geq$ $C_{\varepsilon} q^{\frac{d+1}{2}-\frac{1}{9 d-18}+\varepsilon}$, then $\left|\Delta_{3}(E)\right| \geq c q$ for some $0<c \leq 1$.

The purpose of this paper is to generalize Theorem 1.3. In particular, in the general setting, we improve the third conclusion of Theorem 1.3 by removing the $\varepsilon>0$ in the exponent.

The generalized Erdős-Falconer distance problem has been recently studied by considering the distances between any two sets $E_{1}, E_{2} \subset \mathbb{F}_{q}^{d}$ (see, for example, $[13,16,17,18,22,24,26])$. Given two sets $E_{1}, E_{2} \subset \mathbb{F}_{q}^{d}$, the generalized distance set $\Delta_{2}\left(E_{1}, E_{2}\right)$ is defined by

$$
\Delta_{2}\left(E_{1}, E_{2}\right)=\left\{\left\|\mathbf{x}^{1}-\mathbf{x}^{2}\right\| \in \mathbb{F}_{q}: \mathbf{x}^{1} \in E_{1}, \mathbf{x}^{2} \in E_{2}\right\} .
$$

The generalized Erdős-Falconer distance problem is to determine the smallest exponent $\gamma_{2}>0$ such that if $E_{1}, E_{2} \subset \mathbb{F}_{q}^{d}$ with $\left|E_{1}\right|\left|E_{2}\right| \geq C q^{\gamma_{2}}$ for a sufficiently large constant $C>0$, then $\left|\Delta_{2}\left(E_{1}, E_{2}\right)\right| \geq c q$ for some $0<c \leq 1$. From the ErdősFalconer distance conjecture, it is natural to conjecture that the smallest exponent $\gamma_{2}$ is $d+1$ for odd dimension $d \geq 3$ and $d$ for even dimension $d \geq 2$. Shparlinski [22] obtained the exponent $d+1$ for all dimensions $d \geq 2$. Thus, the generalized Erdős-Falconer distance conjecture was established in odd dimensions. On the other hand, the conjectured exponent $d$ in even dimensions has not been obtained. The currently best known result is the exponent $d+1$ for even dimensions $d$ except for two dimensions. In dimension two the best known result is the exponent $8 / 3$ due to Koh and Shen [16]. 
We now consider a problem which extends both the generalized Erdős-Falconer distance problem and the $k$-resultant modulus set problem. For $k$ sets $E_{j} \subset \mathbb{F}_{q}^{d}, j=$ $1,2, \ldots, k$, we define the generalized $k$-resultant set $\Delta_{k}\left(E_{1}, \ldots, E_{k}\right)$ as

$$
\Delta_{k}\left(E_{1}, \ldots, E_{k}\right)=\left\{\left\|\mathbf{x}^{1}+\mathrm{x}^{2}+\cdots+\mathrm{x}^{k}\right\| \in \mathbb{F}_{q}: \mathbf{x}^{j} \in E_{j}, j=1,2, \ldots, k\right\} .
$$

Problem 1.4. Let $k \geq 2$ be an integer. Suppose that $E_{j} \subset \mathbb{F}_{q}^{d}, j=1,2, \ldots, k$. Determine the smallest exponent $\gamma_{k}>0$ such that if $\prod_{j=1}^{k}\left|E_{j}\right| \geq C q^{\gamma_{k}}$ for a sufficiently large constant $C>0$, then $\left|\Delta_{k}\left(E_{1}, E_{2}, \ldots, E_{k}\right)\right| \geq c q$ for some $0<c \leq 1$.

We call this problem the generalized $k$-resultant modulus problem. As in the $k$-resultant modulus problem, we are only interested in studying this problem in even dimensions $d \geq 2$. If $q=p^{2}$ for some odd prime $p$, then $\mathbb{F}_{q}$ contains the subfield $\mathbb{F}_{p}$. In this case, if the dimension $d$ is even and $E_{j}=\mathbb{F}_{p}^{d}$ for all $j=1,2, \ldots, k$, then $\prod_{j=1}^{k}\left|E_{j}\right|=q^{d k / 2}$ and $\left|\Delta_{k}\left(E_{1}, E_{2}, \ldots, E_{k}\right)\right|=\left|\mathbb{F}_{p}\right|=p=\sqrt{q}$. This example proposes the following conjecture.

Conjecture 1.5. Suppose that $d \geq 2$ is even and $k \geq 2$ is an integer. Then the smallest exponent $\gamma_{k}$ in Problem 1.4 must be $\frac{\mathrm{kd}}{2}$.

1.1. Statement of the main result. As mentioned before, the known results on the generalized Erdős-Falconer distance problem says that if $k=2$, then $2 \leq \gamma_{k} \leq$ $8 / 3$ for $d=2$, and $d \leq \gamma_{k} \leq d+1$ for even dimensions $d \geq 4$, where $\gamma_{k}$ denotes the smallest exponent in Problem 1.4. In this paper we study the generalized $k$ resultant modulus problem for $k \geq 3$. Our main result is as follows.

Theorem 1.6. Let $k \geq 3$ be an integer and $E_{j} \subset \mathbb{F}_{q}^{d}$ for $j=1,2, \ldots, k$. Assume that $C$ is a sufficiently large constant. Then the following statements hold:

(1) If $d=4$ or 6 , and $\prod_{j=1}^{3}\left|E_{j}\right| \geq C q^{3\left(\frac{d+1}{2}-\frac{1}{6 d+2}\right)}$, then $\left|\Delta_{3}\left(E_{1}, E_{2}, E_{3}\right)\right| \gtrsim q$.

(2) If $d \geq 8$ is even and $\prod_{j=1}^{4}\left|E_{j}\right| \geq C q^{4\left(\frac{d+1}{2}-\frac{1}{6 d+2}\right)}$, then $\left|\Delta_{4}\left(E_{1}, E_{2}, E_{3}, E_{4}\right)\right| \gtrsim q$.

(3) If $d \geq 8$ is even and $\prod_{j=1}^{3}\left|E_{j}\right| \geq C q^{3\left(\frac{d+1}{2}-\frac{1}{9 d-18}\right)}$, then $\left|\Delta_{3}\left(E_{1}, E_{2}, E_{3}\right)\right| \gtrsim q$.

Taking $E_{j}=E \subset \mathbb{F}_{q}^{d}$ for all $j=1,2, \ldots, k$, the first and second conclusions of Theorem 1.3 follow immediately from (1),(2) of Theorem 1.6, respectively. Moreover, the third conclusion of Theorem 1.6 implies that the $\varepsilon$ in the statement (3) of Theorem 1.3 is not necessary.

Theorem 1.6 also implies the following result.

Corollary 1.7. For any integer $k \geq 4$, let $E_{j} \subset \mathbb{F}_{q}^{d}$ for $j=1,2, \ldots, k$. Assume that $C>0$ is a sufficiently large constant. Then if $d \geq 4$ is even and $\prod_{j=1}^{k}\left|E_{j}\right| \geq$ $C q^{k\left(\frac{d+1}{2}-\frac{1}{6 d+2}\right)}$, we have $\left|\Delta_{k}\left(E_{1}, E_{2}, \ldots, E_{k}\right)\right| \gtrsim q$.

Proof. Without loss of generality, we may assume that $\left|E_{1}\right| \geq\left|E_{2}\right| \geq \cdots \geq\left|E_{k}\right|$. Notice that if $\prod_{j=1}^{k}\left|E_{j}\right| \geq C q^{k\left(\frac{d+1}{2}-\frac{1}{6 d+2}\right)}$, then $\prod_{j=1}^{k-1}\left|E_{j}\right| \gtrsim q^{(k-1)\left(\frac{d+1}{2}-\frac{1}{6 d+2}\right)}$. Since 
$\left|\Delta_{k-1}\left(E_{1}, \ldots, E_{k-1}\right)\right| \leq\left|\Delta_{k}\left(E_{1}, \ldots, E_{k}\right)\right|$, the statement follows by induction argument with conclusions (1), (2) of Theorem 1.6.

\section{Discrete Fourier ANALYSis}

We shall use the discrete Fourier analysis to deduce the result of our main theorem, Theorem 1.6. In this section, we recall notation and basic concept in the discrete Fourier analysis. Throughout this paper, we shall denote by $\chi$ a nontrivial additive character of $\mathbb{F}_{q}$. Since our result is independent of the choice of the character $\chi$, we assume that $\chi$ is always a fixed nontrivial additive character of $\mathbb{F}_{q}$. The orthogonality relation of $\chi$ states that

$$
\sum_{\mathbf{x} \in \mathbb{F}_{q}^{d}} \chi(\mathbf{m} \cdot \mathbf{x})= \begin{cases}0 & \text { if } \mathbf{m} \neq(0, \ldots, 0) \\ q^{d} & \text { if } \mathbf{m}=(0, \ldots, 0),\end{cases}
$$

where $\mathbf{m} \cdot \mathbf{x}:=\sum_{j=1}^{d} \mathbf{m}_{j} \mathbf{x}_{j}$ for $\mathbf{m}=\left(\mathbf{m}_{1}, \ldots, \mathbf{m}_{d}\right), \mathbf{x}=\left(\mathbf{x}_{1}, \ldots, \mathbf{x}_{d}\right) \in \mathbb{F}_{q}^{d}$. Given a function $g: \mathbb{F}_{q}^{d} \rightarrow \mathbb{C}$, we shall denote by $\widetilde{g}$ the Fourier transform of $g$ which is defined by

$$
\widetilde{g}(\mathbf{x})=\sum_{\mathbf{m} \in \mathbb{F}_{q}^{d}} g(\mathbf{m}) \chi(-\mathbf{x} \cdot \mathbf{m}) \quad \text { for } \mathbf{x} \in \mathbb{F}_{q}^{d}
$$

On the other hand, we shall denote by $\widehat{f}$ the normalized Fourier transform of the function $f: \mathbb{F}_{q}^{d} \rightarrow \mathbb{C}$. Namely we define that

$$
\widehat{f}(\mathbf{m})=\frac{1}{q^{d}} \sum_{\mathbf{x} \in \mathbb{F}_{q}^{d}} f(\mathbf{x}) \chi(-\mathbf{x} \cdot \mathbf{m}) \quad \text { for } \mathbf{m} \in \mathbb{F}_{q}^{d} .
$$

In particular, if $\mathbf{m}=(0, \ldots, 0)$ and we take $f$ as an indicator function of a set $E \subset \mathbb{F}_{q}^{d}$, then we see that

$$
\widehat{E}(0, \ldots, 0)=\frac{|E|}{q^{d}}
$$

Here, and throughout this paper, we write $E(\mathbf{x})$ for the indicator function $1_{E}(\mathbf{x})$ of a set $E \subset \mathbb{F}_{q}^{d}$. We define the normalized inverse Fourier transform of $f$, denoted by $f^{\vee}$, as $f^{\vee}(\mathbf{m})=\widehat{f}(-\mathbf{m})$ for $\mathbf{m} \in \mathbb{F}_{q}^{d}$. It is not hard to see that $\widetilde{\left(f^{\vee}\right)}(\mathbf{x})=f(\mathbf{x})$ for $\mathbf{x} \in \mathbb{F}_{q}^{d}$. Hence, we obtain the Fourier inversion theorem:

$$
f(\mathbf{x})=\sum_{\mathbf{m} \in \mathbb{F}_{q}^{d}} \widehat{f}(\mathbf{m}) \chi(\mathbf{m} \cdot \mathbf{x}) \quad \text { for } \mathbf{x} \in \mathbb{F}_{q}^{d} .
$$

Using the orthogonality relation of the character $\chi$, we see that

$$
\sum_{\mathbf{m} \in \mathbb{F}_{q}^{d}}|\widehat{f}(\mathbf{m})|^{2}=\frac{1}{q^{d}} \sum_{\mathbf{x} \in \mathbb{F}_{q}^{d}}|f(\mathbf{x})|^{2} .
$$

We shall call this formula the Plancherel theorem. Notice that if we take $f$ as an indicator function of a set $E \subset \mathbb{F}_{q}^{d}$, then the Plancherel theorem yields that

$$
\sum_{\mathbf{m} \in \mathbb{F}_{q}^{d}}|\widehat{E}(\mathbf{m})|^{2}=\frac{|E|}{q^{d}} .
$$


Recall from Hölder's inequality that if $f_{1}, f_{2}: \mathbb{F}_{q}^{d} \rightarrow \mathbb{C}$, then we have

$$
\sum_{\mathbf{m} \in \mathbb{F}_{q}^{d}}\left|f_{1}(\mathbf{m})\right|\left|f_{2}(\mathbf{m})\right| \leq\left(\sum_{\mathbf{m} \in \mathbb{F}_{q}^{d}}\left|f_{1}(\mathbf{m})\right|^{p_{1}}\right)^{\frac{1}{p_{1}}}\left(\sum_{\mathbf{m} \in \mathbb{F}_{q}^{d}}\left|f_{2}(\mathbf{m})\right|^{p_{2}}\right)^{\frac{1}{p_{2}}},
$$

where $1<p_{1}, p_{2}<\infty$ and $1 / p_{1}+1 / p_{2}=1$. Applying Hölder's inequality repeatedly, we see that if $f_{i}: D \subset \mathbb{F}_{q}^{d} \rightarrow \mathbb{C}$ and $1 \leq p_{i}<\infty$ for $i=1,2, \ldots, k$ with $\sum_{i=1}^{k} \frac{1}{p_{i}}=1$, then

$$
\sum_{\mathbf{m} \in D}\left(\prod_{i=1}^{k}\left|f_{i}(\mathbf{m})\right|\right) \leq \prod_{i=1}^{k}\left(\sum_{\mathbf{m} \in D}\left|f_{i}(\mathbf{m})\right|^{p_{i}}\right)^{\frac{1}{p_{i}}} .
$$

We refer to this formula as the generalized Hölder's inequality.

Lemma 2.1. Let $k \geq 2$ be an integer. If $E_{j} \subset \mathbb{F}_{q}^{d}$ for all $j=1,2, \ldots, k$, then we have

$$
\sum_{\mathbf{m} \in \mathbb{F}_{q}^{d}}\left(\prod_{j=1}^{k}\left|\widehat{E_{j}}(\mathbf{m})\right|\right) \leq q^{-d k+d}\left(\prod_{j=1}^{k}\left|E_{j}\right|\right)^{\frac{k-1}{k}}
$$

Proof. Notice that if $E \subset \mathbb{F}_{q}^{d}$ and $\mathbf{m} \in \mathbb{F}_{q}^{d}$, then $|\widehat{E}(\mathbf{m})| \leq|\widehat{E}(0, \ldots, 0)|=\frac{|E|}{q^{d}}$. Since $\sum_{j=1}^{k} \frac{1}{k}=1$, applying generalized Hölder's inequality yields the desirable result:

$$
\begin{aligned}
\sum_{\mathbf{m} \in \mathbb{F}_{q}^{d}}\left(\prod_{j=1}^{k}\left|\widehat{E_{j}}(\mathbf{m})\right|\right) & \leq \prod_{j=1}^{k}\left(\sum_{\mathbf{m} \in \mathbb{F}_{q}^{d}}\left|\widehat{E_{j}}(\mathbf{m})\right|^{k}\right)^{\frac{1}{k}} \\
& \leq \prod_{j=1}^{k}\left(\left|\widehat{E_{j}}(0, \ldots, 0)\right|^{\frac{k-2}{k}}\left(\sum_{\mathbf{m} \in \mathbb{F}_{q}^{d}}\left|\widehat{E_{j}}(\mathbf{m})\right|^{2}\right)^{\frac{1}{k}}\right) \\
& =\prod_{j=1}^{k}\left(\left(\frac{\left|E_{j}\right|}{q^{d}}\right)^{\frac{k-2}{k}}\left(\frac{\left|E_{j}\right|}{q^{d}}\right)^{\frac{1}{k}}\right)=q^{-d k+d}\left(\prod_{j=1}^{k}\left|E_{j}\right|\right)^{\frac{k-1}{k}}
\end{aligned}
$$

To estimate a lower bound of $\left|\Delta_{k}\left(E_{1}, \ldots, E_{k}\right)\right|$, we shall utilize the Fourier decay estimate on spheres. Recall that the sphere $S_{t} \subset \mathbb{F}_{q}^{d}$ for $t \in \mathbb{F}_{q}$ is defined by

$$
S_{t}=\left\{\mathbf{x} \in \mathbb{F}_{q}^{d}: x_{1}^{2}+\cdots+x_{d}^{2}=t\right\} .
$$

It is not hard to see that $\left|S_{t}\right|=q^{d-1}(1+o(1))$ for $d \geq 3$ and $t \in \mathbb{F}_{q}$ (see Theorem 6.26 and Theorem 6.27 in [20]). It is well known that the value of $\widehat{S}_{t}(\mathbf{m})$ can be written in terms of the Gauss sum and the Kloosterman sum. In particular, when the dimension $d$ is even, the following result can be obtained from Lemma 4 in [14].

Lemma 2.2. Let $d \geq 2$ be even. If $t \in \mathbb{F}_{q}$ and $\boldsymbol{m} \in \mathbb{F}_{q}^{d}$, then we have

$$
\widehat{S}_{t}(\boldsymbol{m})=q^{-1} \delta_{0}(\boldsymbol{m})+q^{-d-1} G^{d} \sum_{\ell \in \mathbb{F}_{q}^{*}} \chi\left(t \ell+\frac{\|\boldsymbol{m}\|}{4 \ell}\right),
$$


where $\delta_{0}(\boldsymbol{m})=1$ for $\boldsymbol{m}=(0, \ldots, 0)$ and $\delta_{0}(\boldsymbol{m})=0$ otherwise, and $G$ denotes the Gauss sum

$$
G=\sum_{s \in \mathbb{F}_{q}^{*}} \eta(s) \chi(s)
$$

where $\eta$ is the quadratic character of $\mathbb{F}_{q}$, and $\mathbb{F}_{q}^{*}=\mathbb{F}_{q} \backslash\{0\}$. In particular, we have

$$
\widehat{S_{0}}(\boldsymbol{m})=q^{-1} \delta_{0}(\boldsymbol{m})+q^{-d-1} G^{d} \sum_{\ell \in \mathbb{F}_{q}^{*}} \chi(\|\boldsymbol{m}\| \ell) \quad \text { for } \boldsymbol{m} \in \mathbb{F}_{q}^{d} .
$$

We shall invoke the following result which was given in Proposition 2.2 in [18].

Lemma 2.3. If $\boldsymbol{m}, \boldsymbol{v} \in \mathbb{F}_{q}^{d}$, then we have

$$
\left.\sum_{t \in \mathbb{F}_{q}} \widehat{S}_{t}(\boldsymbol{m}){\widehat{S_{t}}}_{(} \boldsymbol{v}\right)=q^{-1} \delta_{0}(\boldsymbol{m}) \delta_{0}(\boldsymbol{v})+q^{-d-1} \sum_{s \in \mathbb{F}_{q}^{*}} \chi(s(\|\boldsymbol{m}\|-\|\boldsymbol{v}\|)) .
$$

3. Formula for a LOWER Bound of $\left|\Delta_{k}\left(E_{1}, \ldots, E_{k}\right)\right|$

This section devotes to proving the following result which is useful to deduce a lower bound of $\left|\Delta_{k}\left(E_{1}, \ldots, E_{k}\right)\right|$.

Theorem 3.1. Let $d \geq 2$ be even and $k \geq 2$ be an integer. If $E_{j} \subset \mathbb{F}_{q}^{d}$ for $j=1,2, \ldots, k$ and $\prod_{j=1}^{k}\left|E_{j}\right| \geq 3{ }^{k} q^{\frac{d k}{2}}$, then we have

$$
\left|\Delta_{k}\left(E_{1}, \ldots, E_{k}\right)\right| \gtrsim \min \left\{q, \frac{\left(\prod_{j=1}^{k}\left|E_{j}\right|\right)^{\frac{k+1}{k}}}{q^{d k}\left(\max _{r \in \mathbb{F}_{q}^{*}} \prod_{j=1}^{k}\left(\sum_{\mathbf{v} \in S_{r}}\left|\widehat{E_{j}}(\mathbf{v})\right|^{k}\right)^{\frac{1}{k}}\right)}\right\} .
$$

Proof. For each $t \in \mathbb{F}_{q}$, we define a counting function $\nu_{k}(t)$ by

$$
\nu_{k}(t)=\left|\left\{\left(\mathbf{x}^{1}, \mathbf{x}^{2}, \ldots, \mathbf{x}^{k}\right) \in E_{1} \times \ldots \times E_{k}:\left\|\mathbf{x}^{1}+\cdots+\mathbf{x}^{k}\right\|=t\right\}\right| .
$$

Since $\prod_{j=1}^{k}\left|E_{j}\right|=\sum_{t \in \mathbb{F}_{q}} \nu_{k}(t)$ and $\nu_{k}(t)=0$ for $t \notin \Delta_{k}\left(E_{1}, \ldots, E_{k}\right)$, we see that

$$
\prod_{j=1}^{k}\left|E_{j}\right|-\nu_{k}(0)=\sum_{0 \neq t \in \Delta_{k}\left(E_{1}, \ldots, E_{k}\right)} \nu_{k}(t) .
$$

Square both sides of this equation and use the Cauchy-Schwarz inequality. It follows

$$
\left(\prod_{j=1}^{k}\left|E_{j}\right|-\nu_{k}(0)\right)^{2}=\left(\sum_{0 \neq t \in \Delta_{k}\left(E_{1}, \ldots, E_{k}\right)} \nu_{k}(t)\right)^{2} \leq\left|\Delta_{k}\left(E_{1}, \ldots, E_{k}\right)\right|\left(\sum_{t \in \mathbb{F}_{q}^{*}} \nu_{k}^{2}(t)\right) .
$$

Thus, we obtain

$$
\left|\Delta_{k}\left(E_{1}, \ldots, E_{k}\right)\right| \geq \frac{\left(\prod_{j=1}^{k}\left|E_{j}\right|-\nu_{k}(0)\right)^{2}}{\sum_{t \in \mathbb{F}_{q}^{*}} \nu_{k}^{2}(t)}
$$


Now, we claim three facts below.

Claim 3.2. Suppose that $d \geq 2$ is even and $k \geq 2$ is an integer. If $E_{j} \subset \mathbb{F}_{q}^{d}$ for $j=1,2, \ldots, k$ with $\prod_{j=1}^{k}\left|E_{j}\right| \geq 3^{k} q^{\frac{d k}{2}}$, then we have

$$
\left(\prod_{j=1}^{k}\left|E_{j}\right|-\nu_{k}(0)\right)^{2} \geq \frac{1}{9}\left(\prod_{j=1}^{k}\left|E_{j}\right|\right)^{2} .
$$

Claim 3.3. Let $d \geq 2$ and $k \geq 2$ be integers. If $E_{j} \subset \mathbb{F}_{q}^{d}$ for $j=1,2, \ldots, k$, then we have

$$
\sum_{t \in \mathbb{F}_{q}} \nu_{k}^{2}(t) \leq \frac{1}{q}\left(\prod_{j=1}^{k}\left|E_{j}\right|\right)^{2}+q^{2 d k-d} \sum_{r \in \mathbb{F}_{q}}\left|\sum_{\mathbf{v} \in S_{r}}\left(\prod_{j=1}^{k} \widehat{E_{j}}(\mathbf{v})\right)\right|^{2} .
$$

Claim 3.4. Assume that $d \geq 2$ is even and $k \geq 2$ is an integer. If $E_{j} \subset \mathbb{F}_{q}^{d}$ for $j=1,2, \ldots, k$ with $\prod_{j=1}^{k}\left|E_{j}\right| \geq q^{\frac{d k}{2}}$, then we have

$$
q^{2 d k-d}\left|\sum_{\mathbf{m} \in S_{0}}\left(\prod_{j=1}^{k} \widehat{E_{j}}(\mathbf{m})\right)\right|^{2}-\nu_{k}^{2}(0) \leq \frac{4}{q}\left(\prod_{j=1}^{k}\left|E_{j}\right|\right)^{2} .
$$

For a moment, let us accept Claims 3.2, 3.3, and 3.4 which shall be proved in the following subsections (see Subsections 3.1, 3.2, and 3.3). From (3.1) and Claim 3.2 , we see that if $\prod_{j=1}^{k}\left|E_{j}\right| \geq 3^{k} q^{\frac{d k}{2}}$, then

$$
\left|\Delta_{k}\left(E_{1}, \ldots, E_{k}\right)\right| \gtrsim \frac{\left(\prod_{j=1}^{k}\left|E_{j}\right|\right)^{2}}{\sum_{t \in \mathbb{F}_{q}^{*}} \nu_{k}^{2}(t)} .
$$

Observe from Claims 3.3 and 3.4 that if $\prod_{j=1}^{k}\left|E_{j}\right| \geq 3^{k} q^{\frac{d k}{2}}$, then

$$
\begin{aligned}
\sum_{t \in \mathbb{F}_{q}^{*}} \nu_{k}^{2}(t) & =\sum_{t \in \mathbb{F}_{q}} \nu_{k}^{2}(t)-\nu_{k}^{2}(0) \leq \frac{5}{q}\left(\prod_{j=1}^{k}\left|E_{j}\right|\right)^{2}+q^{2 d k-d} \sum_{r \neq 0}\left|\sum_{\mathbf{v} \in S_{r}}\left(\prod_{j=1}^{k} \widehat{E_{j}}(\mathbf{v})\right)\right|^{2} \\
& \lesssim \frac{1}{q}\left(\prod_{j=1}^{k}\left|E_{j}\right|\right)^{2}+q^{2 d k-d}\left(\max _{r \neq 0} \sum_{\mathbf{v} \in S_{r}}\left(\prod_{j=1}^{k}\left|\widehat{E_{j}}(\mathbf{v})\right|\right)\right)\left(\sum_{\mathbf{v} \in \mathbb{F}_{q}^{d}}\left(\prod_{j=1}^{k}\left|\widehat{E_{j}}(\mathbf{v})\right|\right)\right) \\
& \leq \frac{1}{q}\left(\prod_{j=1}^{k}\left|E_{j}\right|\right)^{2}+q^{d k}\left(\prod_{j=1}^{k}\left|E_{j}\right|\right)^{\frac{k-1}{k}}\left(\max _{r \neq 0} \sum_{\mathbf{v} \in S_{r}}\left(\prod_{j=1}^{k}\left|\widehat{E_{j}}(\mathbf{v})\right|\right)\right)
\end{aligned}
$$


where Lemma 2.1 was used to obtain the last inequality. From this estimate and (3.2), it follows that

$$
\begin{aligned}
&\left|\Delta_{k}\left(E_{1}, \ldots, E_{k}\right)\right| \gtrsim \frac{\left(\prod_{j=1}^{k}\left|E_{j}\right|\right)^{2}}{\frac{1}{q}\left(\prod_{j=1}^{k}\left|E_{j}\right|\right)^{2}+q^{d k}\left(\prod_{j=1}^{k}\left|E_{j}\right|\right)^{\frac{k-1}{k}}\left(\max _{r \in \mathbb{F}_{q}^{*}} \sum_{\mathbf{v} \in S_{r}}\left(\prod_{j=1}^{k}\left|\widehat{E_{j}}(\mathbf{v})\right|\right)\right)} \\
& \gtrsim \min \left\{q, \frac{\left(\prod_{j=1}^{k}\left|E_{j}\right|\right)^{\frac{k+1}{k}}}{q^{d k}\left(\max _{r \in \mathbb{F}_{q}^{*}} \sum_{\mathbf{v} \in S_{r}}\left(\prod_{j=1}^{k}\left|\widehat{E_{j}}(\mathbf{v})\right|\right)\right)}\right\}
\end{aligned}
$$

Then the statement of Theorem 3.1 follows by applying generalized Hölder's inequality (2.2):

$$
\max _{r \in \mathbb{F}_{q}^{*}} \sum_{\mathbf{v} \in S_{r}}\left(\prod_{j=1}^{k}\left|\widehat{E_{j}}(\mathbf{v})\right|\right) \leq \max _{r \in \mathbb{F}_{q}^{*}} \prod_{j=1}^{k}\left(\sum_{\mathbf{v} \in S_{r}}\left|\widehat{E_{j}}(\mathbf{v})\right|^{k}\right)^{\frac{1}{k}} .
$$

3.1. Proof of Claim 3.2. Suppose that $d \geq 2$ is even and $k \geq 2$ is an integer. Let $E_{j} \subset \mathbb{F}_{q}^{d}$ for $j=1,2, \ldots, k$ with $\prod_{j=1}^{k}\left|E_{j}\right| \geq 3^{k} q^{\frac{d k}{2}}$. We aim to show that

$$
\left(\prod_{j=1}^{k}\left|E_{j}\right|-\nu_{k}(0)\right)^{2} \geq \frac{1}{9}\left(\prod_{j=1}^{k}\left|E_{j}\right|\right)^{2}
$$

To prove this, we begin by estimating the counting function $\nu_{k}(t)$ for $t \in \mathbb{F}_{q}$. For each $t \in \mathbb{F}_{q}$, it follows that

$$
\begin{aligned}
\nu_{k}(t) & =\left|\left\{\left(\mathbf{x}^{1}, \mathbf{x}^{2}, \ldots, \mathbf{x}^{k}\right) \in E_{1} \times \ldots \times E_{k}:\left\|\mathbf{x}^{1}+\cdots+\mathbf{x}^{k}\right\|=t\right\}\right| \\
& =\sum_{\left(\mathbf{x}^{1}, \mathbf{x}^{2}, \ldots, \mathbf{x}^{k}\right) \in E_{1} \times E_{2} \times \cdots \times E_{k}} S_{t}\left(\mathbf{x}^{1}+\mathbf{x}^{2}+\cdots+\mathbf{x}^{k}\right) .
\end{aligned}
$$

Applying the Fourier inversion theorem to the indicate function $S_{t}\left(\mathbf{x}^{1}+\mathbf{x}^{2}+\cdots+\mathbf{x}^{k}\right)$, it follows that

$$
\nu_{k}(t)=\sum_{\left(\mathbf{x}^{1}, \mathbf{x}^{2}, \ldots, \mathbf{x}^{k}\right) \in \mathbb{F}_{q}^{d} \times \cdots \times \mathbb{F}_{q}^{d}} E_{1}\left(\mathbf{x}^{1}\right) \cdots E_{k}\left(\mathbf{x}^{k}\right) \sum_{\mathbf{m} \in \mathbb{F}_{q}^{d}} \widehat{S}_{t}(\mathbf{m}) \chi\left(\mathbf{m} \cdot\left(\mathbf{x}^{1}+\cdots+\mathbf{x}^{k}\right)\right) .
$$

By the definition of the normalized Fourier transform, we can write

$$
\nu_{k}(t)=q^{d k} \sum_{\mathbf{m} \in \mathbb{F}_{q}^{d}} \widehat{S}_{t}(\mathbf{m})\left(\prod_{j=1}^{k} \overline{\widehat{E}_{j}}(\mathbf{m})\right)
$$


To prove (3.3), we first find an upper bound of $\nu_{k}(0)=q^{d k} \sum_{\mathbf{m} \in \mathbb{F}_{q}^{d}} \widehat{S_{0}}(\mathbf{m})\left(\prod_{j=1}^{k} \widehat{\widehat{E_{j}}}(\mathbf{m})\right)$.

By (2.4) of Lemma 2.2, we can write

$$
\begin{aligned}
\nu_{k}(0) & =q^{d k} \sum_{\mathbf{m} \in \mathbb{F}_{q}^{d}}\left(\prod_{j=1}^{k} \overline{\bar{E}_{j}}(\mathbf{m})\right)\left(q^{-1} \delta_{0}(\mathbf{m})+q^{-d-1} G^{d} \sum_{\ell \in \mathbb{F}_{q}^{*}} \chi(\|\mathbf{m}\| \ell)\right) \\
& =q^{d k-1}\left(\prod_{j=1}^{k} \widehat{\widehat{E_{j}}}(0, \ldots, 0)\right)+q^{d k-d-1} G^{d} \sum_{\mathbf{m} \in \mathbb{F}_{q}^{d}}\left(\prod_{j=1}^{k} \widehat{\widehat{E}_{j}}(\mathbf{m})\right) \sum_{\ell \in \mathbb{F}_{q}^{*}} \chi(\|\mathbf{m}\| \ell)
\end{aligned}
$$

Since $\widehat{\widehat{E_{j}}}(0, \ldots, 0)=\frac{\left|E_{j}\right|}{q^{d}},|G|=q^{1 / 2}$, and $\left|\sum_{\ell \in \mathbb{F}_{q}^{*}} \chi(\|\mathbf{m}\| \ell)\right| \leq q$, it follows that

$$
\nu_{k}(0) \leq q^{-1} \prod_{j=1}^{k}\left|E_{j}\right|+q^{d k-d / 2} \sum_{\mathbf{m} \in \mathbb{F}_{q}^{d}} \prod_{j=1}^{k}\left|\widehat{E_{j}}(\mathbf{m})\right|
$$

Applying Lemma 2.1,

$$
\nu_{k}(0) \leq q^{-1} \prod_{j=1}^{k}\left|E_{j}\right|+q^{\frac{d}{2}}\left(\prod_{j=1}^{k}\left|E_{j}\right|\right)^{\frac{k-1}{k}} .
$$

Since $q \geq 3$, it follows that

$$
\begin{aligned}
\prod_{j=1}^{k}\left|E_{j}\right|-\nu_{k}(0) & \geq \prod_{j=1}^{k}\left|E_{j}\right|-q^{-1} \prod_{j=1}^{k}\left|E_{j}\right|-q^{\frac{d}{2}}\left(\prod_{j=1}^{k}\left|E_{j}\right|\right)^{\frac{k-1}{k}} \\
& \geq \frac{1}{3} \prod_{j=1}^{k}\left|E_{j}\right|+\left(\frac{1}{3} \prod_{j=1}^{k}\left|E_{j}\right|-q^{\frac{d}{2}}\left(\prod_{j=1}^{k}\left|E_{j}\right|\right)^{\frac{k-1}{k}}\right) .
\end{aligned}
$$

Note that if $\prod_{j=1}^{k}\left|E_{j}\right| \geq 3^{k} q^{\frac{d k}{2}}$, then the second term above is nonnegative. Thus we obtain that

$$
\prod_{j=1}^{k}\left|E_{j}\right|-\nu_{k}(0) \geq \frac{1}{3} \prod_{j=1}^{k}\left|E_{j}\right| .
$$

Squaring the both sizes, we complete the proof of Claim 3.2.

3.2. Proof of Claim 3.3. We want to prove the following $L^{2}$ estimate of the counting function $\nu(t)$ :

$$
\sum_{t \in \mathbb{F}_{q}} \nu_{k}^{2}(t) \leq \frac{1}{q}\left(\prod_{j=1}^{k}\left|E_{j}\right|\right)^{2}+q^{2 d k-d} \sum_{r \in \mathbb{F}_{q}}\left|\sum_{\mathbf{v} \in S_{r}}\left(\prod_{j=1}^{k} \widehat{E_{j}}(\mathbf{v})\right)\right|^{2} .
$$


By (3.4), we see that

$$
\begin{aligned}
\sum_{t \in \mathbb{F}_{q}} \nu_{k}^{2}(t) & =\sum_{t \in \mathbb{F}_{q}} \nu_{k}(t) \overline{\nu_{k}}(t) \\
& =q^{2 d k} \sum_{\mathbf{m}, \mathbf{v} \in \mathbb{F}_{q}^{d}}\left(\prod_{j=1}^{k} \overline{\widehat{E}_{j}}(\mathbf{m})\right)\left(\prod_{j=1}^{k} \widehat{E_{j}}(\mathbf{v})\right) \sum_{t \in \mathbb{F}_{q}} \widehat{S}_{t}(\mathbf{m}) \overline{\widehat{S}_{t}}(\mathbf{v})
\end{aligned}
$$

Using Lemma 2.3, we see that

$$
\begin{aligned}
& \sum_{t \in \mathbb{F}_{q}} \nu_{k}^{2}(t)=q^{2 d k-1} \sum_{\mathbf{m}, \mathbf{v} \in \mathbb{F}_{q}^{d}}\left(\prod_{j=1}^{k} \overline{\widehat{E_{j}}}(\mathbf{m})\right)\left(\prod_{j=1}^{k} \widehat{E_{j}}(\mathbf{v})\right) \delta_{0}(\mathbf{m}) \delta_{0}(\mathbf{v}) \\
& +q^{2 d k-d-1} \sum_{\mathbf{m}, \mathbf{v} \in \mathbb{F}_{q}^{d}}\left(\prod_{j=1}^{k} \overline{E_{j}}(\mathbf{m})\right)\left(\prod_{j=1}^{k} \widehat{E_{j}}(\mathbf{v})\right)\left(\sum_{s \in \mathbb{F}_{q}} \chi(s(\|\mathbf{m}\|-\|\mathbf{v}\|))-1\right) \\
& =q^{2 d k-1}\left(\prod_{j=1}^{k} \widehat{\widehat{E_{j}}}(0, \ldots, 0)\right)\left(\prod_{j=1}^{k} \widehat{E_{j}}(0, \ldots, 0)\right) \\
& +q^{2 d k-d-1} \sum_{\mathbf{m}, \mathbf{v} \in \mathbb{F}_{q}^{d}}\left(\prod_{j=1}^{k} \overline{\widehat{E_{j}}}(\mathbf{m})\right)\left(\prod_{j=1}^{k} \widehat{E_{j}}(\mathbf{v})\right) \sum_{s \in \mathbb{F}_{q}} \chi(s(\|\mathbf{m}\|-\|\mathbf{v}\|)) \\
& -q^{2 d k-d-1} \sum_{\mathbf{m}, \mathbf{v} \in \mathbb{F}_{q}^{d}}\left(\prod_{j=1}^{k} \overline{\bar{E}_{j}}(\mathbf{m})\right)\left(\prod_{j=1}^{k} \widehat{E_{j}}(\mathbf{v})\right) \text {. }
\end{aligned}
$$

By the definition of the normalized Fourier transform, the orthogonality relation of $\chi$, and basic property of summation, it follows that

$$
\begin{aligned}
& \sum_{t \in \mathbb{F}_{q}} \nu_{k}^{2}(t)=q^{2 d k-1}\left(\prod_{j=1}^{k} \frac{\left|E_{j}\right|}{q^{d}}\right)^{2} \\
& +q^{2 d k-d} \sum_{\mathbf{m}, \mathbf{v} \in \mathbb{F}_{q}^{d}:\|\mathbf{m}\|=\|\mathbf{v}\|}\left(\prod_{j=1}^{k} \widehat{\widehat{E_{j}}}(\mathbf{m})\right)\left(\prod_{j=1}^{k} \widehat{E_{j}}(\mathbf{v})\right) \\
& -q^{2 d k-d-1}\left|\sum_{\mathbf{v} \in \mathbb{F}_{q}^{d}}\left(\prod_{j=1}^{k} \widehat{E_{j}}(\mathbf{v})\right)\right|^{2}
\end{aligned}
$$

Since the third term above is not positive, we obtain that

$$
\begin{aligned}
\sum_{t \in \mathbb{F}_{q}} \nu_{k}^{2}(t) & \leq q^{2 d k-1}\left(\prod_{j=1}^{k} \frac{\left|E_{j}\right|}{q^{d}}\right)^{2}+q^{2 d k-d} \sum_{\mathbf{m}, \mathbf{v} \in \mathbb{F}_{q}^{d}:\|\mathbf{m}\|=\|\mathbf{v}\|}\left(\prod_{j=1}^{k} \widehat{\widehat{E_{j}}}(\mathbf{m})\right)\left(\prod_{j=1}^{k} \widehat{E_{j}}(\mathbf{v})\right) \\
& =q^{-1}\left(\prod_{j=1}^{k}\left|E_{j}\right|\right)^{2}+q^{2 d k-d} \sum_{r \in \mathbb{F}_{q}}\left|\sum_{\mathbf{v} \in \mathbb{F}_{q}^{d}:\|\mathbf{v}\|=r}\left(\prod_{j=1}^{k} \widehat{E_{j}}(\mathbf{v})\right)\right|^{2},
\end{aligned}
$$

which completes the proof of Claim 3.3. 
3.3. Proof of Claim 3.4. For even $d \geq 2$ and an integer $k \geq 2$, let $E_{j} \subset \mathbb{F}_{q}^{d}$ for $j=1,2, \ldots, k$ with $\prod_{j=1}^{k}\left|E_{j}\right| \geq q^{\frac{d k}{2}}$. We must show that

$$
q^{2 d k-d}\left|\sum_{\mathbf{m} \in S_{0}}\left(\prod_{j=1}^{k} \widehat{E_{j}}(\mathbf{m})\right)\right|^{2}-\nu_{k}^{2}(0) \leq \frac{4}{q}\left(\prod_{j=1}^{k}\left|E_{j}\right|\right)^{2} .
$$

We begin by recalling from (3.5) that if $d \geq 2$ is even, then

$$
\nu_{k}(0)=q^{d k-1}\left(\prod_{j=1}^{k} \overline{\widehat{E_{j}}}(0, \ldots, 0)\right)+q^{d k-d-1} G^{d} \sum_{\mathbf{m} \in \mathbb{F}_{q}^{d}}\left(\prod_{j=1}^{k} \overline{\widehat{E_{j}}}(\mathbf{m})\right) \sum_{\ell \in \mathbb{F}_{q}^{*}} \chi(\|\mathbf{m}\| \ell) .
$$

It follows that

$$
\begin{aligned}
\nu_{k}(0)= & q^{-1}\left(\prod_{j=1}^{k}\left|E_{j}\right|\right)+q^{d k-d-1} G^{d} \sum_{\mathbf{m} \in \mathbb{F}_{q}^{d}}\left(\prod_{j=1}^{k} \overline{\widehat{E}_{j}}(\mathbf{m})\right)\left(-1+\sum_{\ell \in \mathbb{F}_{q}} \chi(\|\mathbf{m}\| \ell)\right) \\
= & {\left[q^{-1}\left(\prod_{j=1}^{k}\left|E_{j}\right|\right)-q^{d k-d-1} G^{d} \sum_{\mathbf{m} \in \mathbb{F}_{q}^{d}}\left(\prod_{j=1}^{k} \overline{\bar{E}_{j}}(\mathbf{m})\right)\right] } \\
& +q^{d k-d} G^{d} \sum_{\mathbf{m} \in S_{0}}\left(\prod_{j=1}^{k} \widehat{\widehat{E_{j}}}(\mathbf{m})\right):=\mathrm{A}+\mathrm{B} .
\end{aligned}
$$

Thus we can write

$$
\nu_{k}^{2}(0)=\nu_{k}(0) \overline{\nu \nu_{k}(0)}=(\mathrm{A}+\mathrm{B})(\overline{\mathrm{A}}+\overline{\mathrm{B}})=|\mathrm{A}|^{2}+|\mathrm{B}|^{2}+\mathrm{A} \overline{\mathrm{B}}+\overline{\mathrm{A}} \mathrm{B}
$$

Since the absolute value of the Gauss sum $G$ is $\sqrt{q}$, we have

$$
\nu_{k}^{2}(0)=q^{2 d k-d}\left|\sum_{\mathbf{m} \in S_{0}}\left(\prod_{j=1}^{k} \widehat{E_{j}}(\mathbf{m})\right)\right|^{2}+|\mathrm{A}|^{2}+\mathrm{A} \overline{\mathrm{B}}+\overline{\mathrm{A}} \mathrm{B} .
$$

It follows that

$$
q^{2 d k-d}\left|\sum_{\mathbf{m} \in S_{0}}\left(\prod_{j=1}^{k} \widehat{E_{j}}(\mathbf{m})\right)\right|^{2}-\nu_{k}^{2}(0) \leq-\mathrm{A} \overline{\mathrm{B}}-\overline{\mathrm{A}} \mathrm{B} \leq 2|\mathrm{~A}||\mathrm{B}| .
$$

Now, notice that

$$
|A| \leq q^{-1}\left(\prod_{j=1}^{k}\left|E_{j}\right|\right)+q^{d k-d-1}|G|^{d} \sum_{\mathbf{m} \in \mathbb{F}_{q}^{d}}\left(\prod_{j=1}^{k}\left|\widehat{E_{j}}(\mathbf{m})\right|\right)
$$

and

$$
|B| \leq q^{d k-d}|G|^{d} \sum_{\mathbf{m} \in \mathbb{F}_{q}^{d}}\left(\prod_{j=1}^{k}\left|\widehat{E_{j}}(\mathbf{m})\right|\right)
$$


Since $|G|=\sqrt{q}$, using Lemma 2.1 yields the following two estimates:

$$
|A| \leq q^{-1}\left(\prod_{j=1}^{k}\left|E_{j}\right|\right)+q^{\frac{d}{2}-1}\left(\prod_{j=1}^{k}\left|E_{j}\right|\right)^{\frac{k-1}{k}}
$$

and

$$
|B| \leq q^{\frac{d}{2}}\left(\prod_{j=1}^{k}\left|E_{j}\right|\right)^{\frac{k-1}{k}} .
$$

From these estimates and (3.8), we have

$$
\begin{aligned}
& q^{2 d k-d}\left|\sum_{\mathbf{m} \in S_{0}}\left(\prod_{j=1}^{k} \widehat{E_{j}}(\mathbf{m})\right)\right|^{2}-\nu_{k}^{2}(0) \\
& \leq 2\left(q^{\frac{d}{2}-1}\left(\prod_{j=1}^{k}\left|E_{j}\right|\right)^{\frac{2 k-1}{k}}+q^{d-1}\left(\prod_{j=1}^{k}\left|E_{j}\right|\right)^{\frac{2 k-2}{k}}\right) .
\end{aligned}
$$

Finally, we obtain the estimate (3.7) by observing that if $\prod_{j=1}^{k}\left|E_{j}\right| \geq q^{\frac{d k}{2}}$, then

$$
\max \left\{q^{\frac{d}{2}-1}\left(\prod_{j=1}^{k}\left|E_{j}\right|\right)^{\frac{2 k-1}{k}}, q^{d-1}\left(\prod_{j=1}^{k}\left|E_{j}\right|\right)^{\frac{2 k-2}{k}}\right\} \leq q^{-1}\left(\prod_{j=1}^{k}\left|E_{j}\right|\right)^{2} .
$$

Thus the proof of Claim 3.4 is complete.

\section{CONNECTION BETWEEN RESTRICTION ESTIMATES FOR SPHERES AND}

$$
\left|\Delta_{k}\left(E_{1}, \ldots, E_{k}\right)\right|
$$

Theorem 3.1 shows that a good lower bound of $\left|\Delta_{k}\left(E_{1}, E_{2}, \ldots, E_{k}\right)\right|$ can be obtained by estimating an upper bound of the quantity

$$
\max _{r \in \mathbb{F}_{q}^{*}} \prod_{j=1}^{k}\left(\sum_{\mathbf{v} \in S_{r}}\left|\widehat{E_{j}}(\mathbf{v})\right|^{k}\right)^{\frac{1}{k}} \text {. }
$$

This quantity is closely related to the restriction estimates for spheres with non-zero radius. In this section, we review the restriction problem for spheres and we restate Theorem 3.1 in terms of the restriction estimates for spheres. We begin by reviewing the extension problem for spheres which is also called the dual restriction problem for spheres. We shall use the notation $\left(\mathbb{F}_{q}^{d}, d \mathbf{x}\right)$ to denote the $d$-dimensional vector space over the finite field $\mathbb{F}_{q}$ where a normalized counting measure $d \mathbf{x}$ is given. On the other hand, we denote by $\left(\mathbb{F}_{q}^{d}, d \mathbf{m}\right)$ the dual space of the vector space $\left(\mathbb{F}_{q}^{d}, d \mathbf{x}\right)$,

where we endow the dual space $\left(\mathbb{F}_{q}^{d}, d \mathbf{m}\right)$ with the counting measure $d \mathbf{m}$. Since the space $\left(\mathbb{F}_{q}^{d}, d \mathbf{x}\right)$ can be identified with its dual space $\left(\mathbb{F}_{q}^{d}, d \mathbf{x}\right)$ as an abstract group, we shall use the notation $\mathbb{F}_{q}^{d}$ to indicate both the space and its dual space. To distinguish the space with its dual space, we always use the variable $\mathbf{x}$ for the element of the space $\left(\mathbb{F}_{q}^{d}, d \mathbf{x}\right)$ with the normalized counting measure $d \mathbf{x}$. On the other hand, the variable $\mathbf{m}$ will be used to denote the element of the dual space 
$\left(\mathbb{F}_{q}^{d}, d \mathbf{m}\right)$ with the counting measure $d \mathbf{m}$. For example, we write $\mathbf{x} \in \mathbb{F}_{q}^{d}$ and $\mathbf{m} \in \mathbb{F}_{q}^{d}$ for $\mathbf{x} \in\left(\mathbb{F}_{q}^{d}, d \mathbf{x}\right)$ and $\mathbf{m} \in\left(\mathbb{F}_{q}^{d}, d \mathbf{m}\right)$, respectively. With these notations, the classical norm notation can be used to indicate the following sums: for $1 \leq r<\infty$,

$$
\begin{aligned}
& \|g\|_{L^{r}\left(\mathbb{F}_{q}^{d}, d \mathbf{m}\right)}^{r}=\sum_{\mathbf{m} \in \mathbb{F}_{q}^{d}}|g(\mathbf{m})|^{r}, \\
& \|f\|_{L^{r}\left(\mathbb{F}_{q}^{d}, d \mathbf{x}\right)}^{r}=q^{-d} \sum_{\mathbf{x} \in \mathbb{F}_{q}^{d}}|f(\mathbf{x})|^{r},
\end{aligned}
$$

and

$$
\|g\|_{L^{\infty}\left(\mathbb{F}_{q}^{d}, d \mathbf{m}\right)}=\max _{\mathbf{m} \in \mathbb{F}_{q}^{d}}|g(\mathbf{m})| .
$$

where $g$ is a function on $\left(\mathbb{F}_{q}^{d}, d \mathbf{m}\right)$ and $f$ is a function on $\left(\mathbb{F}_{q}^{d}, d \mathbf{x}\right)$. For each $t \in \mathbb{F}_{q}^{*}$, let $S_{t} \subset\left(\mathbb{F}_{q}^{d}, d x\right)$ be the sphere defined as in (2.3). We endow the sphere $S_{t}$ with the normalized surface measure $d \sigma$ which is defined by measuring the mass of each point on $S_{t}$ as $1 /\left|S_{t}\right|$. Notice that the total mass of $S_{t}$ is 1 and we have

$$
\begin{aligned}
\|f\|_{L^{r}\left(S_{t}, d \sigma\right)}^{r} & =\frac{1}{\left|S_{t}\right|} \sum_{\mathbf{x} \in S_{t}}|f(\mathbf{x})|^{r} \quad \text { for } 1 \leq r<\infty, \\
\|f\|_{L^{\infty}\left(S_{t}, d \sigma\right)} & =\max _{\mathbf{x} \in S_{t}}|f(\mathbf{x})| .
\end{aligned}
$$

We also recall that if $f:\left(S_{t}, d \sigma\right) \rightarrow \mathbb{C}$, then the inverse Fourier transform of $f d \sigma$ is defined by

$$
(f d \sigma)^{\vee}(\mathbf{m})=\frac{1}{\left|S_{t}\right|} \sum_{\mathbf{x} \in S_{t}} f(\mathbf{x}) \chi(\mathbf{m} \cdot \mathbf{x}) \quad \text { for } \mathbf{m} \in\left(\mathbb{F}_{q}^{d}, d \mathbf{m}\right) .
$$

Since the sphere $S_{t}$ is symmetric about the origin, we can write

$$
(d \sigma)^{\vee}(\mathbf{m})=\frac{q^{d}}{\left|S_{t}\right|} \widehat{S}_{t}(\mathbf{m}) \quad \text { for } \mathbf{m} \in\left(\mathbb{F}_{q}^{d}, d \mathbf{m}\right) .
$$

With the above notation, the extension problem for the sphere $S_{t}$ asks us to determine $1 \leq p, r \leq \infty$ such that there exists $C>0$ satisfying the following extension estimate:

$$
\left\|(f d \sigma)^{\vee}\right\|_{L^{r}\left(\mathbb{F}_{q}^{d}, d \mathbf{m}\right)} \leq C\|f\|_{L^{p}\left(S_{t}, d \sigma\right)} \text { for all } f: S_{t} \rightarrow \mathbb{C},
$$

where the constant $C>0$ may depend on $p, r, d, S_{t}$, but it must be independent of the functions $f$ and the size of the underlying finite field $\mathbb{F}_{q}$. By duality, this extension estimate is the same as the following restriction estimate (see $[19,23]$ ) :

$$
\|\widetilde{g}\|_{L^{p^{\prime}\left(S_{t}, d \sigma\right)}} \leq C\|g\|_{L^{r^{\prime}\left(\mathbb{F}_{q}^{d}, d \mathbf{m}\right)}} \text { for all } g: \mathbb{F}_{q}^{d} \rightarrow \mathbb{C},
$$

where $\widetilde{g}$ is defined as in (2.1) and $p^{\prime}, r^{\prime}$ denote the Hölder conjugates of $p$ and $r$, respectively (namely, $1 / p+1 / p^{\prime}=1$ and $1 / r+1 / r^{\prime}=1$ ).

Now, we address the relation between the restriction estimates for spheres with non-zero radius and a lower bound of $\left|\Delta_{k}\left(E_{1}, \ldots, E_{k}\right)\right|$. By Theorem 3.1 and the definition of the restriction estimates for spheres in (4.4), we obtain the following result. 
Lemma 4.1. For even $d \geq 2$ and an integer $k \geq 2$, let $E_{j} \subset \mathbb{F}_{q}^{d}$ for $j=1,2, \ldots, k$. Assume that $\prod_{j=1}^{k}\left|E_{j}\right| \geq 3^{k} q^{\frac{d k}{2}}$ and the following restriction estimate holds for some $1 \leq \ell<\infty$ and $\alpha \in \mathbb{R}$ :

$$
\left\|\widetilde{E_{j}}\right\|_{L^{k}\left(S_{r}, d \sigma\right)} \lesssim q^{\alpha}\left\|E_{j}\right\|_{L^{\ell}\left(\mathbb{F}_{q}^{d}, d m\right)} \quad \text { for all } r \in \mathbb{F}_{q}^{*}, j=1,2, \ldots, k .
$$

Then we have

$$
\left|\Delta_{k}\left(E_{1}, \ldots, E_{k}\right)\right| \gtrsim \min \left\{q, \frac{\left(\prod_{j=1}^{k}\left|E_{j}\right|\right)^{\frac{k+1}{k}-\frac{1}{\ell}}}{q^{k \alpha+d-1}}\right\} .
$$

Proof. By Theorem 3.1, it suffices to prove that

$$
q^{d k}\left(\max _{r \in \mathbb{F}_{q}^{*}} \prod_{j=1}^{k}\left(\sum_{\mathbf{v} \in S_{r}}\left|\widehat{E_{j}}(\mathbf{v})\right|^{k}\right)^{\frac{1}{k}}\right) \lesssim q^{k \alpha+d-1}\left(\prod_{j=1}^{k}\left|E_{j}\right|\right)^{\frac{1}{\ell}} .
$$

Since $\widehat{E_{j}}(\mathbf{v})=q^{-d} \widetilde{E_{j}}(\mathbf{v})$ for $j=1, \ldots, k$, we see that

$$
q^{d k}\left(\max _{r \in \mathbb{F}_{q}^{*}} \prod_{j=1}^{k}\left(\sum_{\mathbf{v} \in S_{r}}\left|\widehat{E_{j}}(\mathbf{v})\right|^{k}\right)^{\frac{1}{k}}\right)=\left(\max _{r \in \mathbb{F}_{q}^{*}} \prod_{j=1}^{k}\left(\sum_{\mathbf{v} \in S_{r}}\left|\widetilde{E_{j}}(\mathbf{v})\right|^{k}\right)^{\frac{1}{k}}\right) .
$$

Using the definition of $\left\|\widetilde{E_{j}}\right\|_{L^{k}\left(S_{r}, d \sigma\right)}$ in (4.2) and the fact that $\left|S_{r}\right| \sim q^{d-1}$, the above quantity is similar to the following value:

$$
q^{d-1}\left(\max _{r \in \mathbb{F}_{q}^{*}} \prod_{j=1}^{k}\left\|\widetilde{E_{j}}\right\|_{L^{k}\left(S_{r}, d \sigma\right)}\right) .
$$

By assumption (4.5), this can be dominated by

$$
q^{d-1}\left(\prod_{j=1}^{k}\left(q^{\alpha}\left\|E_{j}\right\|_{L^{\ell}\left(\mathbb{F}_{q}^{d}, d \mathbf{m}\right)}\right)\right)=q^{k \alpha+d-1}\left(\prod_{j=1}^{k}\left|E_{j}\right|^{\frac{1}{\ell}}\right)=q^{k \alpha+d-1}\left(\prod_{j=1}^{k}\left|E_{j}\right|\right)^{\frac{1}{\ell}} .
$$

Putting all estimates together yields the inequality (4.6), which completes the proof.

\section{RESTRICTION THEOREMS FOR SPHERES}

We see from Lemma 4.1 that the restriction estimates for spheres play an important role in determining lower bounds of the cardinality of the generalized $k$ resultant set $\Delta_{k}\left(E_{1}, \ldots, E_{k}\right)$. In particular, our main result (Theorem 1.6) will be proved by making an effort on finding possibly large exponent $\ell \geq 1$ such that the restriction inequality (4.5) holds for $k=3$ or $k=4$. In this section, we shall obtain such restriction estimates. To this end, we shall apply the following dual restriction estimate for spheres with non-zero radius due to the authors in [14].

Lemma 5.1 ([14], Theorem 1). If $d \geq 4$ be even, then

$$
\left\|(F d \sigma)^{\vee}\right\|_{L^{4}\left(\mathbb{F}_{q}^{d}, d m\right)} \lesssim\|F\|_{L^{(12 d-8) /(9 d-12)}\left(S_{t}, d \sigma\right)} \quad \text { for all } F \subset S_{t}, t \neq 0 .
$$


To obtain a restriction estimate for spheres, we shall use the dual estimate of (5.1). To this end, it is useful to review Lorentz spaces in our setting. For a function $f:\left(S_{t}, d \sigma\right) \rightarrow \mathbb{C}$, we denote by $d_{f}$ the distribution function on $[0, \infty)$ :

$$
d_{f}(a):=\frac{1}{\left|S_{t}\right|}\left|\left\{\mathbf{x} \in S_{t}:|f(\mathbf{x})|>a\right\}\right| .
$$

We see that for $1 \leq r \leq \infty$,

$$
\|f\|_{L^{r}\left(S_{t}, d \sigma\right)}^{r}=r \int_{0}^{\infty} s^{r-1} d_{f}(s) d s .
$$

The function $f^{*}$ is defined on $[0, \infty)$ by

$$
f^{*}(s):=\inf \left\{a>0: d_{f}(a) \leq s\right\} .
$$

For $1 \leq p, r \leq \infty$ and a function $f:\left(S_{t}, d \sigma\right) \rightarrow \mathbb{C}$, define

$$
\|f\|_{L^{p, r}\left(S_{t}, d \sigma\right)}:= \begin{cases}\left(\int_{0}^{\infty}\left(s^{1 / p} f^{*}(s)\right)^{r} \frac{d s}{s}\right)^{1 / r} & \text { for } 1 \leq r<\infty \\ \sup _{s>0} s^{1 / p} f^{*}(s) & \text { for } r=\infty\end{cases}
$$

In particular, we see that

$$
\|f\|_{L^{p, 1}\left(S_{t}, d \sigma\right)}=\int_{0}^{\infty} s^{1 / p-1} f^{*}(s) d s .
$$

It is not hard to see that for $1 \leq p \leq \infty$ and $1 \leq r_{1} \leq r_{2} \leq \infty$,

$$
\|f\|_{L^{p, r_{2}\left(S_{t}, d \sigma\right)}} \lesssim\|f\|_{L^{p, r_{1}\left(S_{t}, d \sigma\right)}} \quad \text { and } \quad\|f\|_{L^{p, p}\left(S_{t}, d \sigma\right)}=\|f\|_{L^{p}\left(S_{t}, d \sigma\right)} .
$$

See [9] for further information about Lorentz spaces. With the above notation, the following fact can be deduced.

Lemma 5.2. Let $d \sigma$ be the normalized surface measure on the sphere $S_{t} \subset\left(\mathbb{F}_{q}^{d}, d \mathbf{x}\right)$. Assume that the estimate

$$
\left\|(F d \sigma)^{\vee}\right\|_{L^{r}\left(\mathbb{F}_{q}^{d}, d \mathbf{m}\right)} \lesssim\|F\|_{L^{p}\left(S_{t}, d \sigma\right)}
$$

holds for all subsets $F$ of $S_{t}$. Then we have

$$
\left\|(f d \sigma)^{\vee}\right\|_{L^{r}\left(\mathbb{F}_{q}^{d}, d \mathbf{m}\right)} \lesssim\|f\|_{L^{p, 1}\left(S_{t}, d \sigma\right)}
$$

for all functions $f:\left(S_{t}, d \sigma\right) \rightarrow \mathbb{C}$.

Proof. Without loss of generality, we may assume that $f$ is a nonnegative simple function given by the form

$$
f=\sum_{j=1}^{N} a_{j} 1_{F_{j}}
$$

where $F_{N} \subset F_{N-1} \subset \cdots \subset F_{2} \subset F_{1}$ and $a_{j}>0$ for all $j=1,2, \ldots, N$. Notice that

$$
f^{*}(s)=\sum_{j=1}^{N} a_{j} 1_{\left[0, \frac{\left|F_{j}\right|}{\mid S_{t}}\right]}(s) .
$$

It follows that

$$
\int_{0}^{\infty} s^{\frac{1}{p}-1} f^{*}(s) d s=\int_{0}^{\infty} s^{\frac{1}{p}-1} \sum_{j=1}^{N} a_{j} 1_{\left[0, \frac{\left|F_{j}\right|}{\mid S_{t}}\right]}(s) d s
$$




$$
=\sum_{j=1}^{N} a_{j} \int_{0}^{\frac{\left|F_{j}\right|}{\left|S_{t}\right|}} s^{\frac{1}{p}-1} d s=p \sum_{j=1}^{N} a_{j}\left(\frac{\left|F_{j}\right|}{\left|S_{t}\right|}\right)^{\frac{1}{p}}=p \sum_{j=1}^{N} a_{j}\left\|F_{j}\right\|_{L^{p}\left(S_{t}, d \sigma\right)} .
$$

Namely, we see that

$$
\int_{0}^{\infty} s^{\frac{1}{p}-1} f^{*}(s) d s \sim \sum_{j=1}^{N} a_{j}\left\|F_{j}\right\|_{L^{p}\left(S_{t}, d \sigma\right)} .
$$

Using this estimate along with (5.3) and the hypothesis (5.2), we see that

$$
\begin{aligned}
\left\|(f d \sigma)^{\vee}\right\|_{L^{r}\left(\mathbb{F}_{q}^{d}, d \mathbf{m}\right)} & \leq \sum_{j=1}^{N} a_{j}\left\|\left(F_{j} d \sigma\right)^{\vee}\right\|_{L^{r}\left(\mathbb{F}_{q}^{d}, d \mathbf{m}\right)} \\
& \lesssim \sum_{j=1}^{N} a_{j}\left\|F_{j}\right\|_{L^{p}\left(S_{t}, d \sigma\right)} \sim \int_{0}^{\infty} s^{\frac{1}{p}-1} f^{*}(s) d s=\|f\|_{L^{p, 1}\left(S_{t}, d \sigma\right)} .
\end{aligned}
$$

Hence, the proof is complete.

We shall invoke the following weak-type restriction estimate.

Lemma 5.3. If $d \geq 4$ is even and we put $r_{0}=(12 d-8) /(3 d+4)$, then the weak-type restriction estimate

$$
\|\widetilde{g}\|_{L^{r_{0}, \infty}\left(S_{t}, d \sigma\right)} \lesssim\|g\|_{L^{\frac{4}{3}\left(\mathbb{F}_{q}^{d}, d m\right)}}
$$

holds for all $t \in \mathbb{F}_{q}^{*}$ and for all functions $g:\left(\mathbb{F}_{q}^{d}, d \boldsymbol{m}\right) \rightarrow \mathbb{C}$.

Proof. Since $r_{0}=(12 d-8) /(3 d+4)$, its dual exponent $r_{0}^{\prime}$ is given by

$$
r_{0}^{\prime}=(12 d-8) /(9 d-12) .
$$

Combining Lemma 5.1 with Lemma 5.2, it follows that

$$
\left\|(f d \sigma)^{\vee}\right\|_{L^{4}\left(\mathbb{F}_{q}^{d}, d \mathbf{m}\right)} \lesssim\|f\|_{L^{r_{0}^{\prime}, 1}\left(S_{t}, d \sigma\right)}
$$

for all functions $f:\left(S_{t}, d \sigma\right) \rightarrow \mathbb{C}$ with $t \in \mathbb{F}_{q}^{*}$. By duality, this estimate is same as (5.4), which completes the proof.

The following restriction estimate will play an important role in proving the third part of Theorem 1.6.

Lemma 5.4. If $E \subset\left(\mathbb{F}_{q}^{d}, d \mathbf{m}\right)$ and $|E| \geq q^{\frac{d-1}{2}}$, then we have

$$
\|\widetilde{E}\|_{L^{2}\left(S_{t}, d \sigma\right)} \lesssim \frac{|E|}{q^{\frac{d-1}{4}}} \quad \text { for all } t \in \mathbb{F}_{q}^{*} .
$$

Proof. Since $\|\widetilde{E}\|_{L^{2}\left(S_{t}, d \sigma\right)}^{2}=\frac{1}{\left|S_{t}\right|} \sum_{\mathbf{x} \in S_{t}}|\widetilde{E}(\mathbf{x})|^{2}$ and $\left|S_{t}\right| \sim q^{d-1}$ for $t \in \mathbb{F}_{q}^{*}$, it is enough to show that if $E \subset \mathbb{F}_{q}^{d}$ with $|E| \geq q^{\frac{d-1}{2}}$, then

$$
\sum_{\mathbf{x} \in S_{t}}|\widetilde{E}(\mathbf{x})|^{2} \lesssim q^{\frac{d-1}{2}}|E|^{2}
$$


Notice from the definition of the Fourier transforms that

$$
\begin{aligned}
\sum_{\mathbf{x} \in S_{t}}|\widetilde{E}(\mathbf{x})|^{2} & =\sum_{\mathbf{x} \in S_{t}} \sum_{\mathbf{m}, \mathbf{m}^{\prime} \in E} \chi\left(-\mathbf{x} \cdot\left(\mathbf{m}-\mathbf{m}^{\prime}\right)\right)=\sum_{\mathbf{m}, \mathbf{m}^{\prime} \in E} q^{d} \widehat{S}_{t}\left(\mathbf{m}-\mathbf{m}^{\prime}\right) \\
& =q^{d}|E| \widehat{S}_{t}(0, \ldots, 0)+\sum_{\mathbf{m}, \mathbf{m}^{\prime} \in E: \mathbf{m} \neq \mathbf{m}^{\prime}} q^{d} \widehat{S}_{t}\left(\mathbf{m}-\mathbf{m}^{\prime}\right) \\
& \leq|E|\left|S_{t}\right|+\left(\max _{\mathbf{n} \in \mathbb{F}_{q}^{d} \backslash\{(0, \ldots, 0)\}}\left|\widehat{S_{t}}(\mathbf{n})\right|\right) \sum_{\mathbf{m}, \mathbf{m}^{\prime} \in E: \mathbf{m}_{\neq} \neq \mathbf{m}^{\prime}} q^{d} \\
& \lesssim|E| q^{d-1}+|E|^{2} q^{d}\left(\max _{\mathbf{n} \in \mathbb{F}_{q}^{d} \backslash\{(0, \ldots, 0)\}}\left|\widehat{S}_{t}(\mathbf{n})\right|\right) .
\end{aligned}
$$

Now, we apply the well known fact (Lemma 2.2 in [15]) that if $S_{t} \subset \mathbb{F}_{q}^{d}$ for $t \in \mathbb{F}_{q}^{*}$ and $d \geq 2$, then

$$
\left(\max _{\mathbf{n} \in \mathbb{F}_{q}^{d} \backslash\{(0, \ldots, 0)\}}\left|\widehat{S}_{t}(\mathbf{n})\right|\right) \lesssim q^{-\frac{d+1}{2}} .
$$

Then we see that

$$
\sum_{\mathbf{x} \in S_{t}}|\widetilde{E}(\mathbf{x})|^{2} \lesssim|E| q^{d-1}+q^{\frac{d-1}{2}}|E|^{2} \lesssim q^{\frac{d-1}{2}}|E|^{2},
$$

where the last inequality follows from our assumption that $|E| \geq q^{\frac{d-1}{2}}$. Thus, (5.5) holds and we complete the proof.

Now, we introduce the interpolation theorem which enables us to derive the restriction estimates we need for the proof of our main results.

Theorem 5.5. Let $\Omega$ be a collection of subsets $E$ of $\left(\mathbb{F}_{q}^{d}, d \mathbf{m}\right)$. Assume that the following two restriction estimates hold for all sets $E \in \Omega$ and $1 \leq r_{0}<r_{1} \leq \infty$ :

$$
\|\widetilde{E}\|_{L^{r_{0}, \infty}\left(S_{t}, d \sigma\right)} \lesssim A_{0}(q,|E|):=A_{0}
$$

and

$$
\|\widetilde{E}\|_{L^{r_{1}, \infty}\left(S_{t}, d \sigma\right)} \lesssim A_{1}(q,|E|):=A_{1} .
$$

Then for $r_{0}<r<r_{1}$, we have

$$
\|\widetilde{E}\|_{L^{r}\left(S_{t}, d \sigma\right)} \lesssim\left(\max \left\{\frac{2 r}{r-r_{0}}, \frac{2 r}{r_{1}-r}\right\}\right)^{\frac{1}{r}} A_{0}^{\frac{r_{0}\left(r_{1}-r\right)}{r\left(r_{1}-r_{0}\right)}} A_{1}^{\frac{r_{1}\left(r-r_{0}\right)}{r\left(r_{1}-r_{0}\right)}} .
$$

Namely, if $\frac{1}{r}=\frac{1-\theta}{r_{0}}+\frac{\theta}{r_{1}}$ for some $0<\theta<1$, then we have

$$
\|\widetilde{E}\|_{L^{r}\left(S_{t}, d \sigma\right)} \lesssim A_{0}^{1-\theta} A_{1}^{\theta} .
$$

Proof. Let $\delta>0$ which will be chosen later.

$$
\begin{gathered}
\|\widetilde{E}\|_{L^{r}\left(S_{t}, d \sigma\right)}^{r}=r \int_{0}^{\infty} s^{r-1} d_{\widetilde{E}}(s) d s \\
=r \int_{0}^{\delta} s^{r-1} d_{\widetilde{E}}(s) d s+r \int_{\delta}^{\infty} s^{r-1} d_{\widetilde{E}}(s) d s \\
=r \int_{0}^{\delta} s^{r-r_{0}-1} s^{r_{0}} d_{\widetilde{E}}(s) d s+r \int_{\delta}^{\infty} s^{r-r_{1}-1} s^{r_{1}} d_{\widetilde{E}}(s) d s
\end{gathered}
$$




$$
\begin{gathered}
\leq r\left(\sup _{0<s<\infty} s^{r_{0}} d_{\widetilde{E}}(s)\right) \int_{0}^{\delta} s^{r-r_{0}-1} d s+r\left(\sup _{0<s<\infty} s^{r_{1}} d_{\widetilde{E}}(s)\right) \int_{\delta}^{\infty} s^{r-r_{1}-1} d s \\
=\frac{r}{r-r_{0}}\|\widetilde{E}\|_{L^{r_{0}, \infty}\left(S_{t}, d \sigma\right)}^{r_{0}} \delta^{r-r_{0}}+\frac{r}{r_{1}-r}\|\widetilde{E}\|_{L^{r_{1}, \infty}\left(S_{t}, d \sigma\right)}^{r_{1}} \delta^{r-r_{1}} \\
\lesssim \max \left\{\frac{r}{r-r_{0}}, \frac{r}{r_{1}-r}\right\}\left(A_{0}^{r_{0}} \delta^{r-r_{0}}+A_{1}^{r_{1}} \delta^{r-r_{1}}\right) .
\end{gathered}
$$

Now we choose $\delta$ such that

$$
A_{0}^{r_{0}} \delta^{r-r_{0}}=A_{1}^{r_{1}} \delta^{r-r_{1}} .
$$

Namely, we choose

$$
\delta=A_{0}^{\frac{r_{0}}{r_{0}-r_{1}}} A_{1}^{\frac{r_{1}}{r_{1}-r_{0}}}
$$

It follows that

$$
\begin{aligned}
\|\widetilde{E}\|_{L^{r}\left(S_{t}, d \sigma\right)}^{r} & \lesssim \max \left\{\frac{2 r}{r-r_{0}}, \frac{2 r}{r_{1}-r}\right\} A_{0}^{r_{0}} \delta^{r-r_{0}} \\
& \lesssim \max \left\{\frac{2 r}{r-r_{0}}, \frac{2 r}{r_{1}-r}\right\} A_{0}^{r_{0}} A_{0}^{\frac{r_{0}\left(r-r_{0}\right)}{r_{0}-r_{1}}} A_{1}^{\frac{r_{1}\left(r-r_{0}\right)}{r_{1}-r_{0}}} \\
& =\max \left\{\frac{2 r}{r-r_{0}}, \frac{2 r}{r_{1}-r}\right\} A_{0}^{\frac{r_{0}\left(r_{1}-r\right)}{r_{1}-r_{0}}} A_{1}^{\frac{r_{1}\left(r-r_{0}\right)}{r_{1}-r_{0}}},
\end{aligned}
$$

which implies (5.6). By a direct computation, (5.7) follows from (5.6).

\section{Proof of main theorem (Theorem 1.6)}

In this section, we shall give the complete proof of Theorem 1.6. Since $\|\widetilde{g}\|_{L^{\infty, \infty}\left(S_{t}, d \sigma\right)}=$ $\|\widetilde{g}\|_{L^{\infty}\left(S_{t}, d \sigma\right)}=\max _{\mathbf{x} \in S_{t}}|\widetilde{g}(\mathbf{x})| \leq\|g\|_{L^{1}\left(\mathbb{F}_{q}^{d}, d \mathbf{m}\right)}$, it is clear that

$$
\|\widetilde{E}\|_{L^{\infty, \infty}\left(S_{t}, d \sigma\right)} \lesssim\|E\|_{L^{1}\left(\mathbb{F}_{q}^{d}, d \mathbf{m}\right)}=|E| \text { for all } E \subset \mathbb{F}_{q}^{d}, t \neq 0 .
$$

On the other hand, it follows from Lemma 5.3 that if $d \geq 4$ is even, then

$$
\|\widetilde{E}\|_{L^{\frac{12 d-8}{3 d+4}, \infty}\left(S_{t}, d \sigma\right)} \lesssim\|E\|_{L^{\frac{4}{3}\left(\mathbb{F}_{q}^{d}, d \mathbf{m}\right)}}=|E|^{\frac{3}{4}} \quad \text { for all } E \subset \mathbb{F}_{q}^{d}, t \neq 0 .
$$

6.1. Proof of statement (1) of Theorem 1.6. We need the following lemma.

Lemma 6.1. If $d=4$ or 6 , then we have

$$
\|\widetilde{E}\|_{L^{3}\left(S_{t}, d \sigma\right)} \lesssim\|E\|_{L^{\frac{9 d+12}{6 d+14}\left(\mathbb{F}_{q}^{d}, d \mathbf{m}\right)}} \quad \text { for all } E \subset \mathbb{F}_{q}^{d}, t \neq 0 .
$$

Proof. Note that if $d=4$ or 6 , then $\frac{12 d-8}{3 d+4}<3<\infty$. Therefore, using Theorem 5.5, we are able to interpolate (6.1) and (6.2) so that we obtain

$$
\|\widetilde{E}\|_{L^{3}\left(S_{t}, d \sigma\right)} \lesssim|E|^{1-\theta}|E|^{\frac{3 \theta}{4}} \quad \text { with } \theta=\frac{12 d-8}{9 d+12} .
$$

Namely, we see that

$$
\|\widetilde{E}\|_{L^{3}\left(S_{t}, d \sigma\right)} \lesssim|E|^{\frac{6 d+14}{9 d+12}}=\|E\|_{L^{\frac{9 d+12}{6 d+14}\left(\mathbb{F}_{q}^{d}, d \mathbf{m}\right)}} .
$$

Thus, the proof is complete. 
We are ready to prove statement (1) of Theorem 1.6. We aim to prove that if $d=4$ or $d=6$, and $E_{1}, E_{2}, E_{3} \subset \mathbb{F}_{q}^{d}$ with $\left|E_{1}\right|\left|E_{2}\right|\left|E_{3}\right| \gtrsim q^{3\left(\frac{d+1}{2}-\frac{1}{6 d+2}\right)}$, then $\left|\Delta_{3}\left(E_{1}, E_{2}, E_{3}\right)\right| \gtrsim q$. Combining Lemma 4.1 and Lemma 6.1 , we see that

$$
\left|\Delta_{3}\left(E_{1}, E_{2}, E_{3}\right)\right| \gtrsim \min \left\{q, \frac{\left(\prod_{j=1}^{3}\left|E_{j}\right|\right)^{\frac{4}{3}-\frac{6 d+14}{9 d+12}}}{q^{d-1}}\right\} .
$$

Since $\left|E_{1}\right|\left|E_{2}\right|\left|E_{3}\right| \geq C q^{3\left(\frac{d+1}{2}-\frac{1}{6 d+2}\right)}$ for a sufficiently large $C>0$, we see from a direct computation that

$$
\left|\Delta_{3}\left(E_{1}, E_{2}, E_{3}\right)\right| \gtrsim q .
$$

6.2. Proof of statement (2) of Theorem 1.6. We shall utilize the following key lemma.

Lemma 6.2. If $d \geq 4$ is even, then we have

$$
\|\widetilde{E}\|_{L^{4}\left(S_{t}, d \sigma\right)} \lesssim\|E\|_{L^{\frac{12 d+16}{9 d+18}\left(\mathbb{F}_{q}^{d}, d \mathbf{m}\right)}} \text { for all } E \subset \mathbb{F}_{q}^{d}, t \neq 0 .
$$

Proof. Since $\frac{12 d-8}{3 d+4}<4<\infty$ for all even $d \geq 4$, interpolating (6.1) and (6.2) yields

$$
\|\widetilde{E}\|_{L^{4}\left(S_{t}, d \sigma\right)} \lesssim|E|^{1-\theta}|E|^{\frac{3 \theta}{4}} \quad \text { with } \theta=\frac{3 d-2}{3 d+4} .
$$

Since $|E|^{1-\theta}|E|^{\frac{3 \theta}{4}}=|E|^{\frac{9 d+18}{12 d+16}}=\|E\|_{L^{\frac{12 d+16}{9 d+18}\left(\mathbb{F}_{q}^{d}, d \mathbf{m}\right)}}$, the statement follows.

Let us prove statement (2) of Theorem 1.6. Recall that we must show that if $d \geq 8$ is even and $E_{1}, E_{2}, E_{3}, E_{4} \subset \mathbb{F}_{q}^{d}$ with $\prod_{j=1}^{4}\left|E_{j}\right| \gtrsim q^{4\left(\frac{d+1}{2}-\frac{1}{6 d+2}\right)}$, then $\left|\Delta_{4}\left(E_{1}, E_{2}, E_{3}, E_{4}\right)\right| \gtrsim q$. Combining Lemma 4.1 and Lemma 6.2, we obtain that

$$
\left|\Delta_{4}\left(E_{1}, E_{2}, E_{3}, E_{4}\right)\right| \gtrsim \min \left\{q, \frac{\left(\prod_{j=1}^{4}\left|E_{j}\right|\right)^{\frac{5}{4}-\frac{9 d+18}{12 d+16}}}{q^{d-1}}\right\} .
$$

Since $\left|E_{1}\right|\left|E_{2}\right|\left|E_{3}\right|\left|E_{4}\right| \geq C q^{4\left(\frac{d+1}{2}-\frac{1}{6 d+2}\right)}$ for a sufficiently large $C>0$, it follows from a direct computation that

$$
\left|\Delta_{4}\left(E_{1}, E_{2}, E_{3}, E_{4}\right)\right| \gtrsim q .
$$

6.3. Proof of statement (3) of Theorem 1.6. We begin by proving the following lemma.

Lemma 6.3. Let $E \subset \mathbb{F}_{q}^{d}$. If $d \geq 8$ is even and $|E| \geq q^{\frac{d-1}{2}}$, then we have

$$
\|\widetilde{E}\|_{L^{3}\left(S_{t}, d \sigma\right)} \lesssim q^{\frac{-3 d^{2}+23 d-20}{36 d-96}}\|E\|_{L^{\frac{18 d-48}{15 d-46}\left(\mathbb{F}_{q}^{d}, d \mathbf{m}\right)}} \quad \text { for all } t \in \mathbb{F}_{q}^{*} .
$$


Proof. Since $\|\widetilde{E}\|_{L^{2, \infty}\left(S_{t}, d \sigma\right)} \leq\|\widetilde{E}\|_{L^{2}\left(S_{t}, d \sigma\right)}$ and $|E| \geq q^{\frac{d-1}{2}}$, we see from Lemma 5.4 that

$$
\|\widetilde{E}\|_{L^{2, \infty}\left(S_{t}, d \sigma\right)} \lesssim q^{-\frac{(d-1)}{4}}|E| \quad \text { for all } t \in \mathbb{F}_{q}^{*} \quad \text { and } E \subset \mathbb{F}_{q}^{d} \text { with }|E| \geq q^{\frac{d-1}{2}}
$$

As in (6.2), we also see that

$$
\|\widetilde{E}\|_{L^{\frac{12 d-8}{3 d+4}, \infty}\left(S_{t}, d \sigma\right)} \lesssim|E|^{\frac{3}{4}} \quad \text { for all } E \subset \mathbb{F}_{q}^{d}, t \in \mathbb{F}_{q}^{*} .
$$

Since $2<3<\frac{12 d-8}{3 d+4}$ for $d \geq 8$, by using Theorem 5.5 we are able to interpolate (6.3) and (6.4). Hence, if $d \geq 8$ is even and $|E| \geq q^{\frac{d-1}{2}}$, then we have

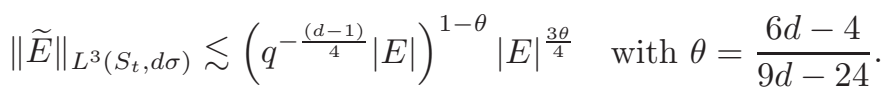

By a direct computation, we conclude

$$
\|\widetilde{E}\|_{L^{3}\left(S_{t}, d \sigma\right)} \lesssim q^{\frac{-3 d^{2}+23 d-20}{36 d-96}}|E|^{\frac{15 d-46}{18 d-48}}=q^{\frac{-3 d^{2}+23 d-20}{36 d-96}}\|E\|_{L^{\frac{18 d-48}{15 d-46}}\left(\mathbb{F}_{q}^{d}, d \mathbf{m}\right)},
$$

which completes the proof of the lemma.

Let us prove the statement (3) of Theorem 1.6 which states that if $d \geq 8$ is even and $\prod_{j=1}^{3}\left|E_{j}\right| \gtrsim q^{3\left(\frac{d+1}{2}-\frac{1}{9 d-18}\right)}$, then $\left|\Delta_{3}\left(E_{1}, E_{2}, E_{3}\right)\right| \gtrsim q$. To prove this, let us first assume that one of $\left|E_{1}\right|,\left|E_{2}\right|,\left|E_{3}\right|$ is less than $q^{\frac{d-1}{2}}$, say that $\left|E_{3}\right|<q^{\frac{d-1}{2}}$. Then by our hypothesis that $\left|E_{1}\right|\left|E_{2}\right|\left|E_{3}\right| \gtrsim q^{3\left(\frac{d+1}{2}-\frac{1}{9 d-18}\right)}$, it must follow that

$$
\left|E_{1}\right|\left|E_{2}\right| \gtrsim q^{-\frac{(d-1)}{2}} q^{3\left(\frac{d+1}{2}-\frac{1}{9 d-18}\right)}=q^{(d+1)+\frac{3 d-7}{3 d-6}}>q^{d+1} .
$$

This implies that $\left|\Delta_{2}\left(E_{1}, E_{2}\right)\right| \gtrsim q$, which was proved by Shparlinski [22]. Thus it is clear that $\left|\Delta_{3}\left(E_{1}, E_{2}, E_{3}\right)\right| \gtrsim q$, because $\left|\Delta_{3}\left(E_{1}, E_{2}, E_{3}\right)\right| \geq\left|\Delta_{2}\left(E_{1}, E_{2}\right)\right|$. For this reason, we may assume that all of $\left|E_{1}\right|,\left|E_{2}\right|,\left|E_{3}\right|$ are greater than or equal to $q^{\frac{d-1}{2}}$ and $\left|E_{1}\right|\left|E_{2}\right|\left|E_{3}\right| \gtrsim q^{3\left(\frac{d+1}{2}-\frac{1}{9 d-18}\right)}$. Combining Lemma 4.1 with Lemma 6.3, we obtain that

$$
\left|\Delta_{3}\left(E_{1}, E_{2}, E_{3}\right)\right| \gtrsim \min \left\{q, \frac{\left(\prod_{j=1}^{3}\left|E_{j}\right|\right)^{\frac{4}{3}-\frac{1}{\ell}}}{q^{3 \alpha+d-1}}\right\}
$$

where we take $\alpha=\frac{-3 d^{2}+23 d-20}{36 d-96}$ and $\ell=\frac{18 d-48}{15 d-46}$. By a direct comparison, it is not hard to see that if $\left|E_{1}\right|\left|E_{2}\right|\left|E_{3}\right| \gtrsim q^{3\left(\frac{d+1}{2}-\frac{1}{9 d-18}\right)}=q^{\frac{9 d^{2}-9 d-20}{6 d-12}}$, then we have $\left|\Delta_{3}\left(E_{1}, E_{2}, E_{3}\right)\right| \gtrsim q$. We have finished the proof of the third part of Theorem 1.6.

\section{REFERENCES}

[1] M. Bennett, D. Hart, A. Iosevich, J. Pakianathan, and M. Rudnev, Group actions and geometric combinatorics in $\mathbb{F}_{q}^{d}$, Forum Math. 29 (2017), no.1, 91-110. 2

[2] M. Bennett, A. Iosevich, and J. Pakianathan, Three-point congurations determined by subsets of $\mathbb{F}_{q}^{2}$ via the Elekes-Sharir Paradigm, Combinatorica, 34 (2014), no.6, 689-706. 2

[3] J. Bourgain, N. Katz, and T. Tao, A sum-product estimate in finite fields, and applications, Geom. Funct. Anal. 14 (2004), 27-57. 1, 2

[4] J. Chapman, M. Erdog̃an, D. Hart, A. Iosevich, and D. Koh, Pinned distance sets, Wolff's exponent in finite fields and sum-product estimates, Math.Z. 271 (2012), 63-93. 2 
[5] D. Covert, D. Koh, and Y. Pi, On the sums of any $k$ points in finite fields, SIAM J. Discrete Math. 30 (2016), no.1, 367-382. 1, 2, 3

[6] D. Covert, D. Koh, and Y. Pi, The k-resultant modulus set problem on algebraic varieties over finite fields, www.arxiv(2015). 3

[7] D. Hart, and A. Iosevich, Ubiquity of simplices in subsets of vector spaces over finite fields, Anal. Math. 34 (2008), no.1, 29-38. 2

[8] P. Erdös, On sets of distances of $n$ points, Amer. Math. Monthly, 53 (1946), 248-250. 1

[9] L. Grafakos, Classical and modern Fourier analysis, Pearson. Education, Inc. (2004). 16

[10] L. Guth and N. Katz, On the Erdös distinct distance problem in the plane, Annals of Mathematics, 181 (2015), 155-190. 1

[11] D. Hart, A. Iosevich, D. Koh and M. Rudnev, Averages over hyperplanes, sum-product theory in vector spaces over finite fields and the Erdös-Falconer distance conjecture, Trans. Amer. Math. Soc. 363 (2011), no.6, 3255-3275. 2

[12] B. Hanson, B. Lund, and O. Roche-Newton, On distinct perpendicular bisectors and pinned distances in finite fields, Finite Fields and Their Applications, 37 (2016), 240-264. 2

[13] D. Hart, L. Li and C. Shen, Fourier analysis and expanding phenomena in finite fields, Proc. Amer. Math. Soc. 141(2013), no.2, 461-473. 3

[14] A. Iosevich and D. Koh, Extension theorems for spheres in the finite field setting, Forum Math. 22 (2010), no.3, 457-483. 6, 15

[15] A. Iosevich and M. Rudnev, Erdös distance problem in vector spaces over finite fields, Trans. Amer. Math. Soc. 359 (2007), 6127-6142. 1, 2, 18

[16] D. Koh and C. Shen, Sharp extension theorems and Falconer distance problems for algebraic curves in two dimensional vector spaces over finite fields, Rev. Mat. Iberoam. 28 (2012), no.1, 157-178. 3

[17] D. Koh and C. Shen, The generalized Erdös-Falconer distance problems in vector spaces over finite fields, J. Number Theory 132 (2012), no.11, 2455-2473. 3

[18] D. Koh and H, Sun, Distance sets of two subsets of vector spaces over finite fields, Proc. Amer. Math. Soc. 143 (2015), no.4, 1679-1692. 3, 7

[19] G. Mockenhaupt, and T. Tao,Restriction and Kakeya phenomena for finite fields, Duke Math. J. 121 (2004), no. 1, 35-74. 14

[20] R. Lidl and H. Niederreiter, Finite fields, Cambridge University Press, (1997). 6

[21] D. H. Pham, T. Pham, and L.A. Vinh, An improvement on the number of simplices in $\mathbb{F}_{q}^{d}$, Discrete Appl. Math. 221 (2017), 95-105. 2

[22] I. Shparlinski, On the set of distance between two sets over finite fields, International Journal of Mathematics and Mathematical Sciences Volume 2006, Article ID 59482, Pages 1-5. 3, 21

[23] T. Tao, Some recent progress on the restriction conjecture, Fourier analysis and convexity, 217-243, Appl. Numer. Harmon. Anal., Birkhäuser Boston, Boston, MA, (2004). 14

[24] L. A. Vinh, Explicit Ramsey graphs and Erdös distance problem over finite Euclidean and non-Euclidean spaces, Electron. J. Combin. 15 (2008), no.1, 5-18. 3

[25] L. A. Vinh, On kaleidoscopic pseudo-randomness of finite Euclidean graphs, Discuss. Math. Graph Theory 32 (2012), no.2, 279-287. 2

[26] L. A. Vinh, On the generalized Erdös distance problems over finite fields, J. Number Theory, 133 (2013), no.9, 2939-2947. 3

Department of Mathematics and Computer Science, University of Missouri-St. Louis, ST. Louis, MO 63121 USA

E-mail address: covertdj@umsl.edu

Department of Mathematics, Chungbuk National University, Cheonguu Chungbuk 28644, KOREA

E-mail address: koh131@chungbuk.ac.kr

Department of Mathematics, Chungbuk National University, Cheonguu Chungbuk 28644, KOREA

E-mail address: pi@chungbuk.ac.kr 